\title{
Curvature properties of some class of warped product manifolds
}

\author{
Ryszard Deszcz, Małgorzata Głogowska, Jan Jełowicki and Georges Zafindratafa \\ Dedicated to the memory of Professor Włodzimierz Waliszewski
}

\begin{abstract}
Warped product manifolds with $\mathrm{p}$-dimensional base, $\mathrm{p}=1,2$, satisfy some curvature conditions of pseudosymmetry type. These conditions are formed from the metric tensor $\mathrm{g}$, the Riemann-Christoffel curvature tensor R, the Ricci tensor $\mathrm{S}$ and the Weyl conformal curvature $\mathrm{C}$ of the considered manifolds. The main result of the paper states that if $\mathrm{p}=2$ and the fibre is a semi-Riemannian space of constant curvature, if $\mathrm{n}$ is greater or equal to 4, then the (0,6)-tensors R.R - Q(S,R) and C.C of such warped products are proportional to the $(0,6)$-tensor $\mathrm{Q}(\mathrm{g}, \mathrm{C})$ and the tensor $\mathrm{C}$ is expressed by a linear combination of some Kulkarni-Nomizu products formed from the tensors $\mathrm{g}$ and $\mathrm{S}$. Thus these curvature conditions satisfy non-conformally flat non-Einstein warped product spacetimes $(\mathrm{p}=2, \mathrm{n}=4)$. We also investigate curvature properties of pseudosymmetry type of quasi-Einstein manifolds. In particular, we obtain some curvature property of the Goedel spacetime 1
\end{abstract}

\section{INTRODUCTION}

Let $g, \nabla, R, S, \kappa$ and $C$ be the metric tensor, the Levi-Civita connection, the RiemannChristoffel curvature tensor, the Ricci tensor, the scalar curvature tensor and the Weyl conformal curvature tensor of a semi-Riemannian manifold $(M, g), n=\operatorname{dim} M \geq 3$, respectively. It is well-known that $(M, g)$ is said to be an Einstein manifold if at every point of $M$ its Ricci tensor $S$ is proportional to the metric tensor $g$, i.e., $S=\frac{\kappa}{n} g$ on $M$ [5]. In particular, if $S=0$ on $M$ then $(M, g)$ is called a Ricci flat manifold. We denote by $\mathcal{U}_{S}$ the set of all points of $(M, g)$ at which $S$ is not proportional to $g$, i.e., $\mathcal{U}_{S}=\left\{x \in M \mid S-\frac{\kappa}{n} g \neq 0\right.$ at $\left.x\right\}$. The manifold $(M, g)$ is said to be a quasi-Einstein manifold if

$$
\operatorname{rank}(S-\alpha g)=1
$$

on $\mathcal{U}_{S}$, where $\alpha$ is some function on $\mathcal{U}_{S}$. In particular, if rank $S=1$ on $\mathcal{U}_{S}$ then $(M, g)$ is called a Ricci-simple manifold [19]. Every warped product manifold $\bar{M} \times_{F} \widetilde{N}$ of an 1-dimensional $(\bar{M}, \bar{g})$ base manifold and a 2 -dimensional manifold $(\tilde{N}, \widetilde{g})$ or an $(n-1)$-dimensional Einstein manifold $(\widetilde{N}, \widetilde{g}), n \geq 4$, with a warping function $F$, is a quasi-Einstein manifold (see, e.g., [12, Section 1]). We mention that quasi-Einstein manifolds arose during the study of exact solutions of the Einstein field equations and the investigation on quasi-umbilical hypersurfaces of conformally flat spaces, see, e.g., 29] and references therein. Quasi-Einstein hypersurfaces in semi-Riemannian spaces of constant curvature were studied among others in: [26, 30, 42, 46, 61,

\footnotetext{
${ }^{1}$ Mathematics Subject Classification (2010): Primary 53B20, 53B25, 53B30, 53B50, 83C15; Secondary $53 \mathrm{C} 25,53 \mathrm{C} 40,53 \mathrm{C} 80$.

Key words and phrases: Einstein manifold, quasi-Einstein manifold, three-dimensional Berger sphere, three-spheres of Kaluza-Klein type, 2-quasi-Einstein manifold, warped product manifold, spacetime, RobertsonWalker spacetime, generalized Robertson-Walker spacetime, Gödel spacetime, general spherically symmetric spacetime, Vaidya spacetime, hypersurface, 2-quasi-umbilical hypersurface, Chen ideal submanifold, pseudosymmetric manifold, manifold with pseudosymmetric Weyl tensor, peudosymmetry type curvature condition.

The first named author is supported by the Université de Valenciennes et du Hainaut-Cambrésis, France and by a grant of the Wrocław University of Environmental and Life Sciences, Poland. The second and third named authors are supported by a grant of the Wrocław University of Environmental and Life Sciences, Poland.
} 
see also [29]. We refer to [12, 43] for recent results on quasi-Einstein manifolds. The semiRiemannian manifold $(M, g), n \geq 3$, is called a 2-quasi-Einstein manifold if

$$
\operatorname{rank}(S-\alpha g) \leq 2
$$

on $\mathcal{U}_{S}$ and $\operatorname{rank}(S-\alpha g)=2$ on some open non-empty subset of $\mathcal{U}_{S}$, where $\alpha$ is some function on $\mathcal{U}_{S}$ (see, e.g., [32, 36]). Every warped product manifold $\bar{M} \times_{F} \widetilde{N}$ of a 2-dimensional base manifold $(\bar{M}, \bar{g})$ and a 2-dimensional manifold $(\tilde{N}, \widetilde{g})$ or an $(n-2)$-dimensional Einstein semiRiemannian manifold $(\tilde{N}, \widetilde{g}), n \geq 5$, with a warping function $F$, satisfies (1.2) (see Theorem 6.1 of this paper). Some exact solutions of the Einstein field equations are non-conformally flat 2-quasi-Einstein manifolds. For instance, the Reissner-Nordstrøm spacetime, as well as the Reissner-Nordstrøm-de Sitter type spacetimes are such manifolds (see, e.g., [69]). It seems that the Reissner-Nordstrøm spacetime is the "oldest" example of a non-conformally flat 2-quasiEinstein warped product manifold. It is easy to see that every 2-quasi-umbilical hypersurface in a semi-Riemannian space of constant curvature is a 2-quasi-Einstein manifold (see, e.g., [36]).

Let $A$ and $B$ be symmetric $(0,2)$-tensors on a semi-Riemannian manifold $(M, g)$. We denote by $A \wedge B$ their Kulkarni-Nomizu tensor. We note that (1.1) holds at a point $x \in \mathcal{U}_{S} \subset M$ if and only if at this point we have $(S-\alpha g) \wedge(S-\alpha g)=0$, i.e.

$$
\frac{1}{2} S \wedge S-\alpha g \wedge S+\alpha^{2} G=0, \quad G=\frac{1}{2} g \wedge g
$$

From (1.3), by a suitable contraction, we get immediately

$$
S^{2}=(\kappa-(n-2) \alpha) S+\alpha((n-1) \alpha-\kappa) g .
$$

For precise definitions of the symbols used here, we refer to Section 2 of this paper (see also [12, 29]). We can write the Weyl conformal curvature tensor $C$ of $(M, g), n \geq 3$, by

$$
C=R-\frac{1}{n-2} g \wedge S+\frac{\kappa}{(n-2)(n-1)} G .
$$

It is well-known that a semi-Riemannian manifold $(M, g), n \geq 4$, is conformally flat if and only if $C=0$ everywhere in $M$. From $C=0$, by (1.5), we get immediately

$$
R=\frac{1}{n-2} g \wedge S-\frac{\kappa}{(n-2)(n-1)} G .
$$

The Robertson-Walker spacetimes, and more generally, warped products of an 1-dimensional manifold and an $(n-1)$-dimensional semi-Riemannian space of constant curvature, $n \geq 4$, are conformally flat quasi-Einstein manifolds (see, e.g., [73, Section 4]). It is obvious that (1.3) and (1.6) yield

$$
R=\frac{1}{2} S \wedge S+\left(\frac{1}{n-2}-\alpha\right) g \wedge S+\left(\alpha^{2}-\frac{\kappa}{(n-2)(n-1)}\right) G
$$

(see, e.g., [28, p. 150]). Thus the curvature tensor $R$ of a conformally flat quasi-Einstein manifold $(M, g), n \geq 4$, is expressed by a linear combination of the tensors: $S \wedge S, g \wedge S$ and $G$. We also can investigate non-conformally flat and non-quasi-Einstein semi-Riemannian manifolds $(M, g), n \geq 4$, whose curvature tensor $R$ is a linear combination of these tensors. 
More precisely, we can investigate semi-Riemannian manifolds $(M, g), n \geq 4$, satisfying on the set $\mathcal{U}_{S} \cap \mathcal{U}_{C} \subset M$ the condition

$$
R=\frac{\phi}{2} S \wedge S+\mu g \wedge S+\eta G
$$

where $\mathcal{U}_{C}$ is the set of all points of $M$ at which $C \neq 0$ and $\phi, \mu$ and $\eta$ are some functions on $\mathcal{U}_{S} \cap \mathcal{U}_{C}$. A semi-Riemannian manifold $(M, g), n \geq 4$, satisfying (1.7) on $\mathcal{U}_{S} \cap \mathcal{U}_{C} \subset M$ is called a Roter type manifold, or Roter manifold, or Roter space [25, 35, 36]. Roter type manifolds and in particular Roter type hypersurfaces in semi-Riemannian spaces of constant curvature were studied in: [25, 28, 31, 32, 35, 42, 47, 51, 52, 59, 60, 68, 69]. In Section 3 we present curvature conditions satisfying by Roter type manifolds. In particular, on every Roter type manifold $(M, g), n \geq 4$, the following relations are satisfied on $\mathcal{U}_{S} \cap \mathcal{U}_{C} \subset M$ :

$$
\begin{aligned}
R \cdot R-Q(S, R) & =L Q(g, C) \\
C \cdot C & =L_{C} Q(g, C) \\
C \cdot R+R \cdot C & =Q(S, C)+\left(L+L_{C}-\frac{1}{(n-2) \phi}\right) Q(g, C), \\
C \cdot R-R \cdot C & =Q(S, C)-\frac{\kappa}{n-1} Q(g, C),
\end{aligned}
$$

where $L=L_{R}+\frac{\mu}{\phi}, L_{C}=L_{R}-\frac{\kappa}{n-1}+\frac{1}{(n-2) \phi}-\frac{\mu}{\phi}$ and $L_{R}=\frac{1}{\phi}\left((n-2)\left(\mu^{2}-\phi \eta\right)-\mu\right)$ (Theorem 3.2 and Proposition 3.3). In [78, Theorem 3.2] (also see [36, Section 4] and [79, Section 4]) it was proved that the curvature tensor $R$ of some hypersurfaces in semi-Riemannian spaces of constant curvature is a linear combination of the tensors: $S \wedge S, g \wedge S, G, g \wedge S^{2}, S \wedge S^{2}$ and $S^{2} \wedge S^{2}$. Precisely, we have on $\mathcal{U}_{S} \cap \mathcal{U}_{C} \subset M$

$$
R=\frac{\phi_{1}}{2} S \wedge S+\phi_{2} g \wedge S+\phi_{3} G+\phi_{4} g \wedge S^{2}+\phi_{5} S \wedge S^{2}+\frac{\phi_{6}}{2} S^{2} \wedge S^{2},
$$

where $\phi_{1}, \phi_{2}, \ldots, \phi_{6}$ are some functions on this set. Evidently, (1.7) is a special case of (1.12). Examples of manifolds satisfying (1.12) are given in [33, Example 2.1], [36, Section 4], [43, Example 4.1], [79, Section 5] and [81, Section 5]. Manifolds satisfying (1.12) were studied in [34, 60, 82, 83].

It is easy to verify that on any semi-Riemannian manifold $(M, g), n \geq 4$, the following identity is satisfied

$$
C \cdot R+R \cdot C=R \cdot R+C \cdot C-\frac{1}{(n-2)^{2}} Q\left(g,-\frac{\kappa}{n-1} g \wedge S+g \wedge S^{2}\right)
$$

(Theorem 3.4(i)). In addition, if (1.8), with some function $L$, holds on $\mathcal{U}_{C} \subset M$ then (1.13) turns into

$$
\begin{aligned}
C \cdot R+R \cdot C= & Q(S, C)+L Q(g, C)+C \cdot C \\
& -\frac{1}{(n-2)^{2}} Q\left(g, \frac{n-2}{2} S \wedge S-\kappa g \wedge S+g \wedge S^{2}\right)
\end{aligned}
$$


(Theorem 3.4(ii)). Moreover, if (1.9), with some functions $L_{C}$, is satisfied on $\mathcal{U}_{C} \subset M$ then (1.14) takes the form

$$
\begin{aligned}
C \cdot R+R \cdot C= & Q(S, C)+\left(L+L_{C}\right) Q(g, C) \\
& -\frac{1}{(n-2)^{2}} Q\left(g, \frac{n-2}{2} S \wedge S-\kappa g \wedge S+g \wedge S^{2}\right)
\end{aligned}
$$

(Theorem 3.4(iii)). We note that if $(M, g)$ is a quasi-Einstein semi-Riemannian manifold satisfying (1.1) then (1.15), by making use of (1.3) and (1.4), turns into

$$
C \cdot R+R \cdot C=Q(S, C)+\left(L+L_{C}\right) Q(g, C),
$$

and in particular, if $(M, g)$ is the Gödel spacetime then (1.16) yields

$$
C \cdot R+R \cdot C=Q(S, C)+\frac{\kappa}{6} Q(g, C)
$$

(Theorem 3.4(iv)-(v)). The conditions (1.8) and (1.9) are also satisfied on some submanifolds isometrically immersed in an Euclidean space, as well as on some hypersurfaces isometrically immersed in a semi-Riemannian space of constant curvature (theorems 3.7-3.9).

In Section 4 we prove that warped product manifolds $\bar{M} \times_{F} \widetilde{N}$ of an 1-dimensional semiRiemannian manifold $(\bar{M}, \bar{g})$ and some $(n-1)$-dimensional semi-Riemannian manifold $(\widetilde{N}, \widetilde{g})$, $n \geq 4$, satisfy (1.8), (1.9) and (1.15) (theorems 4.1-4.3). In particular, we state that the warped product of an 1-dimensional manifold $(\bar{M}, \bar{g})$ and some 3-dimensional Riemannian manifold: the 3-dimensional Berger spheres, the Heisenberg group $\left.N i l_{3}, \widehat{P S L(2,}, \mathbb{R}\right)$ - the universal covering of the Lie group $P S L(2, \mathbb{R})$, the Lie group $S_{0} l_{3}$, a Riemannian manifold isometric to an open part of the 3-dimensional Cartan hypersurface or some three-spheres of Kaluza-Klein type, satisfies (1.8), (1.9) and (1.15) (Theorem 4.2).

In the next section we present results on pseudosymmetric warped product manifolds. In particular, we consider warped products $\bar{M} \times_{F} \widetilde{N}$ of a 2-dimensional semi-Riemannian manifold $(\bar{M}, \bar{g})$ and an $(n-2)$-dimensional semi-Riemannian manifold $(\widetilde{N}, \widetilde{g}), n \geq 4$, with the warping function $F$, assuming that $(\tilde{N}, \widetilde{g})$ is a semi-Riemannian space of constant curvature, when $n \geq 5$. In Theorem 5.3 we present necessary and sufficient condition for such manifold to be pseudosymmetric.

In Section 6 we consider warped products $\bar{M} \times_{F} \tilde{N}$ of a 2-dimensional semi-Riemannian manifold $(\bar{M}, \bar{g})$ and an $(n-2)$-dimensional semi-Riemannian manifold $(\widetilde{N}, \widetilde{g}), n \geq 4$, with the warping function $F$, assuming that $(\widetilde{N}, \widetilde{g})$ is an Einstein semi-Riemannian manifold, when $n \geq 5$. Theorem 6.2 states that on some subset $\mathcal{U}_{S} \cap \mathcal{U}_{C} \subset \bar{M} \times_{F} \widetilde{N}$ (see to that section for details) the tensor $R \cdot S$ is a linear combination of the Tachibana tensors $Q(g, S), Q\left(g, S^{2}\right)$ and $Q\left(S, S^{2}\right)$, i.e.

$$
R \cdot S=\psi_{5} Q(g, S)+\psi_{4} Q\left(g, S^{2}\right)+\psi_{3} Q\left(S, S^{2}\right),
$$

on this set, for some functions $\psi_{3}, \psi_{4}$ and $\psi_{5}$. We mention that recently in [36] it was shown that the tensor $R \cdot S$ of some minimal hypersurfaces in Euclidean spaces has this property (see also [37, 77]). The condition (11.18), by (2.19), turns into

$$
C \cdot S=\psi_{1} Q(g, S)+\psi_{2} Q\left(g, S^{2}\right)+\psi_{3} Q\left(S, S^{2}\right),
$$


where $\psi_{1}=\psi_{5}+\frac{\kappa}{(n-2)(n-1)}$ and $\psi_{2}=\psi_{4}-\frac{1}{n-2}$. Semi-Riemannian manifolds, and in particular, hypersurfaces in semi-Riemannian spaces of constant curvature, satisfying the special cases of (1.19), i.e. $C \cdot S=\psi Q(g, S)$, resp., $C \cdot S=0$, were investigated, among others, in [27, 68, 69], resp., [29, 30, 31, 32, 44, 45, 81.

In the last section we consider warped products $\bar{M} \times_{F} \tilde{N}$ of a 2-dimensional semi-Riemannian manifold $(\bar{M}, \bar{g})$ and an $(n-2)$-dimensional semi-Riemannian manifold $(\widetilde{N}, \widetilde{g}), n \geq 4$, with the warping function $F$, assuming that $(\widetilde{N}, \widetilde{g})$ is a semi-Riemannian space of constant curvature, when $n \geq 5$. In Theorem 7.1(i) we state that (1.8), (1.9) and (1.15) hold on $\mathcal{U}_{C} \subset \bar{M} \times_{F} \tilde{N}$. In Theorem 7.1(ii), under some additional assumption, we state that on some open subset $V \subset \mathcal{U}_{S} \cap \mathcal{U}_{C} \subset \bar{M} \times_{F} \widetilde{N}$ the Weyl tensor $C$ of the considered warped product is a linear combination of the Kulkarni-Nomizu tensors $S \wedge S, g \wedge S, g \wedge S^{2}$ and $G$. Precisely, (7.8) holds on $V$. Evidently, (7.8) by (1.5) turns into (1.12). Thus we have a new family of manifolds satisfying (1.12). On the set $\left(\mathcal{U}_{S} \cap \mathcal{U}_{C}\right) \backslash V$ the Weyl tensor $C$ is a linear combination of the Kulkarni-Nomizu tensors $S \wedge S, g \wedge S$ and $G$. In that section we also present curvature properties of the Vaidya spacetime, as well as of some generalized Vaidya spacetimes: the Vaidya-Kottler, the Vaidya-Reissner-Nordstrøm and the Vaidya-Bonnor spacetime.

\section{Preliminary Results}

Throughout this paper all manifolds are assumed to be connected paracompact manifolds of class $C^{\infty}$. Let $(M, g)$ be an $n$-dimensional, $n \geq 2$, semi-Riemannian manifold and let $\nabla$ be its Levi-Civita connection and $\Xi(M)$ the Lie algebra of vector fields on $M$. We define on $M$ the endomorphisms $X \wedge_{A} Y$ and $\mathcal{R}(X, Y)$ of $\Xi(M)$, respectively, by

$$
\left(X \wedge_{A} Y\right) Z=A(Y, Z) X-A(X, Z) Y, \mathcal{R}(X, Y) Z=\nabla_{X} \nabla_{Y} Z-\nabla_{Y} \nabla_{X} Z-\nabla_{[X, Y]} Z,
$$

where $A$ is a symmetric $(0,2)$-tensor on $M$ and $X, Y, Z \in \Xi(M)$. The Ricci tensor $S$, the Ricci operator $\mathcal{S}$, the tensors $S^{2}$ and $S^{3}$ and the scalar curvature $\kappa$ of $(M, g)$ are defined by $S(X, Y)=\operatorname{tr}\{Z \rightarrow \mathcal{R}(Z, X) Y\}, g(\mathcal{S} X, Y)=S(X, Y), S^{2}(X, Y)=S(\mathcal{S} X, Y), S^{3}(X, Y)=$ $S^{2}(\mathcal{S} X, Y)$ and $\kappa=\operatorname{tr} \mathcal{S}$, respectively. The endomorphism $\mathcal{C}(X, Y)$ is defined by

$$
\mathcal{C}(X, Y) Z=\mathcal{R}(X, Y) Z-\frac{1}{n-2}\left(X \wedge_{g} \mathcal{S} Y+\mathcal{S} X \wedge_{g} Y-\frac{\kappa}{n-1} X \wedge_{g} Y\right) Z
$$

Now the $(0,4)$-tensor $G$, the Riemann-Christoffel curvature tensor $R$ and the Weyl conformal curvature tensor $C$ of $(M, g)$ are defined by $G\left(X_{1}, X_{2}, X_{3}, X_{4}\right)=g\left(\left(X_{1} \wedge_{g} X_{2}\right) X_{3}, X_{4}\right)$ and

$$
R\left(X_{1}, X_{2}, X_{3}, X_{4}\right)=g\left(\mathcal{R}\left(X_{1}, X_{2}\right) X_{3}, X_{4}\right), C\left(X_{1}, X_{2}, X_{3}, X_{4}\right)=g\left(\mathcal{C}\left(X_{1}, X_{2}\right) X_{3}, X_{4}\right),
$$

respectively, where $X_{1}, X_{2}, X_{3}, X_{4} \in \Xi(M)$. Let $\mathcal{B}$ be a tensor field sending any $X, Y \in \Xi(M)$ to a skew-symmetric endomorphism $\mathcal{B}(X, Y)$, and let $B$ be a $(0,4)$-tensor associated with $\mathcal{B}$ by

$$
B\left(X_{1}, X_{2}, X_{3}, X_{4}\right)=g\left(\mathcal{B}\left(X_{1}, X_{2}\right) X_{3}, X_{4}\right) .
$$

The tensor $B$ is said to be a generalized curvature tensor if the following conditions are satisfied

$$
\begin{aligned}
& B\left(X_{1}, X_{2}, X_{3}, X_{4}\right)=B\left(X_{3}, X_{4}, X_{1}, X_{2}\right) \\
& B\left(X_{1}, X_{2}, X_{3}, X_{4}\right)+B\left(X_{3}, X_{1}, X_{2}, X_{4}\right)+B\left(X_{2}, X_{3}, X_{1}, X_{4}\right)=0 .
\end{aligned}
$$

For $\mathcal{B}$ as above, let $B$ be again defined by (2.1). We extend the endomorphism $\mathcal{B}(X, Y)$ to a derivation $\mathcal{B}(X, Y)$. of the algebra of tensor fields on $M$, assuming that it commutes with 
contractions and $\mathcal{B}(X, Y) \cdot f=0$, for any smooth function $f$ on $M$. For a $(0, k)$-tensor field $T, k \geq 1$, we can define the $(0, k+2)$-tensor $B \cdot T$ by

$$
\begin{aligned}
& (B \cdot T)\left(X_{1}, \ldots, X_{k}, X, Y\right)=(\mathcal{B}(X, Y) \cdot T)\left(X_{1}, \ldots, X_{k}\right) \\
= & -T\left(\mathcal{B}(X, Y) X_{1}, X_{2}, \ldots, X_{k}\right)-\cdots-T\left(X_{1}, \ldots, X_{k-1}, \mathcal{B}(X, Y) X_{k}\right) .
\end{aligned}
$$

In addition, if $A$ is a symmetric $(0,2)$-tensor then we define the $(0, k+2)$-tensor $Q(A, T)$ by

$$
\begin{aligned}
& Q(A, T)\left(X_{1}, \ldots, X_{k}, X, Y\right)=\left(X \wedge_{A} Y \cdot T\right)\left(X_{1}, \ldots, X_{k}\right) \\
= & -T\left(\left(X \wedge_{A} Y\right) X_{1}, X_{2}, \ldots, X_{k}\right)-\cdots-T\left(X_{1}, \ldots, X_{k-1},\left(X \wedge_{A} Y\right) X_{k}\right) .
\end{aligned}
$$

The tensor $Q(A, T)$ is called the Tachibana tensor of the tensors $A$ and $T$, or shortly the Tachibana tensor (see, e.g., [28, 31, 33, 36, 43]). For a symmetric $(0,2)$-tensor $E$ and a $(0, k)$ tensor $T, k \geq 2$, we define their Kulkarni-Nomizu product $E \wedge T$ by ([27])

$$
\begin{aligned}
& (E \wedge T)\left(X_{1}, \ldots, X_{4}, Y_{3}, \ldots, Y_{k}\right) \\
= & E\left(X_{1}, X_{4}\right) T\left(X_{2}, X_{3}, Y_{3}, \ldots, Y_{k}\right)+E\left(X_{2}, X_{3}\right) T\left(X_{1}, X_{4}, Y_{3}, \ldots, Y_{k}\right) \\
& -E\left(X_{1}, X_{3}\right) T\left(X_{2}, X_{4}, Y_{3}, \ldots, Y_{k}\right)-E\left(X_{2}, X_{4}\right) T\left(X_{1}, X_{3}, Y_{3}, \ldots, Y_{k}\right) .
\end{aligned}
$$

For instance, the following tensors are generalized curvature tensors: $R, C, G$ and $E \wedge F$, where $E$ and $F$ are symmetric (0,2)-tensors. For a symmetric $(0,2)$-tensor $A$ we define the endomorphism $\mathcal{A}$ and the tensors $A^{2}$ and $A^{3}$ by $g(\mathcal{A} X, Y)=A(X, Y), A^{2}(X, Y)=A(\mathcal{A} X, Y)$ and $A^{3}(X, Y)=A^{2}(\mathcal{A} X, Y)$, respectively. Let $B_{h i j k}, T_{h i j k}$, and $A_{i j}$ be the local components of generalized curvature tensors $B$ and $T$ and a symmetric $(0,2)$-tensor $A$ on $M$, respectively, where $h, i, j, k, l, m, p, q \in\{1,2, \ldots, n\}$. The local components $(B \cdot T)_{h i j k l m}$ and $Q(A, T)_{h i j k l m}$ of the tensors $B \cdot T, Q(A, T), B \cdot A$ and $Q(g, A)$ are the following

$$
\begin{aligned}
(B \cdot T)_{h i j k l m}= & g^{p q}\left(T_{p i j k} B_{q h l m}+T_{h p j k} B_{q i l m}+T_{h i p k} B_{q j l m}+T_{h i j p} B_{q k l m}\right) \\
Q(A, T)_{h i j k l m}= & A_{h l} T_{m i j k}+A_{i l} T_{h m j k}+A_{j l} T_{h i m k}+A_{k l} T_{h i j m} \\
& -A_{h m} T_{l i j k}-A_{i m} T_{h l j k}-A_{j m} T_{h i l k}-A_{k m} T_{h i j l} \\
(B \cdot A)_{h k l m}= & g^{p q}\left(A_{p k} B_{q h l m}+A_{p h} B_{q k l m}\right) \\
Q(g, A)_{h k l m}= & g_{h l} A_{k m}+g_{k l} A_{h m}-g_{h m} A_{k l}-g_{k m} A_{h l} .
\end{aligned}
$$

Lemma 2.1. Let $(M, g), n \geq 3$, be a semi-Riemannian manifold. Let $A$ be a symmetric $(0,2)$ tensor on $M$ such that $\operatorname{rank}(A)=2$ at some point $x \in M$. (i) cf. [25, Lemma 2.1] The tensors $A, A^{2}$ and $A^{3}$ satisfy at $x$ the following relations

$$
\begin{aligned}
A^{3} & =\operatorname{tr}(A) A^{2}+\frac{1}{2}\left(\operatorname{tr}\left(A^{2}\right)-(\operatorname{tr}(A))^{2}\right) A, \\
A \wedge A^{2} & =\frac{1}{2} \operatorname{tr}(A) A \wedge A, \\
A^{2} \wedge A^{2} & =-\frac{1}{2}\left(\operatorname{tr}\left(A^{2}\right)-(\operatorname{tr}(A))^{2}\right) A \wedge A, \\
\left(A^{2}-\operatorname{tr}(A) A\right) \wedge\left(A^{2}-\operatorname{tr}(A) A\right) & =-\frac{1}{2}\left(\operatorname{tr}\left(A^{2}\right)-(\operatorname{tr}(A))^{2}\right) A \wedge A .
\end{aligned}
$$

(ii) Let $T$ be a generalized curvature tensor on $M$ satisfying

$$
T=\frac{\phi_{0}}{2} A \wedge A+\phi_{2} g \wedge A+\phi_{3} G+\phi_{4} g \wedge A^{2}+\phi_{5} A \wedge A^{2}+\frac{\phi_{6}}{2} A^{2} \wedge A^{2},
$$


where $\phi_{0}, \phi_{2}, \ldots, \phi_{6}$ are some functions on $M$. Then at given point $x$ we have

$$
\begin{aligned}
T & =\frac{\phi_{1}}{2} A \wedge A+\phi_{2} g \wedge A+\phi_{3} G+\phi_{4} g \wedge A^{2}, \\
\phi_{1} & =\phi_{0}+\operatorname{tr}(A) \phi_{5}-\frac{1}{2}\left(\operatorname{tr}\left(A^{2}\right)-(\operatorname{tr}(A))^{2}\right) \phi_{6} .
\end{aligned}
$$

Proof. (i) (2.6) and (2.7) were already obtained in [25, eqs. (2.6) and (2.10)]. Further, transvecting equation $(2.10)$ of [25], i.e.

$$
\operatorname{tr}(A)\left(A_{i l} A_{j m}-A_{i m} A_{j l}\right)+A_{j l} A_{i m}^{2}+A_{i m} A_{j l}^{2}-A_{i l} A_{j m}^{2}-A_{j m} A_{i l}^{2}=0,
$$

with $A_{k}^{m}=g^{m s} A_{s k}$ we obtain

$$
A_{i l}^{2} A_{j k}^{2}-A_{i k}^{2} A_{j l}^{2}+A_{i l} A_{j k}^{3}-A_{j l} A_{i k}^{3}=\operatorname{tr}(A)\left(A_{i l} A_{j k}^{2}-A_{j l} A_{i k}^{2}\right),
$$

where $g_{h k}, g^{h k}, A_{h k}, A_{h k}^{2}$ and $A_{h k}^{3}$ are the local components of the tensors $g, g^{-1}, A, A^{2}$ and $A^{3}$, respectively. This, by (2.7), turns into

$$
A_{i l}^{2} A_{j k}^{2}-A_{i k}^{2} A_{j l}^{2}=-\frac{1}{2}\left(\operatorname{tr}\left(A^{2}\right)-(\operatorname{tr}(A))^{2}\right)\left(A_{i l} A_{j k}-A_{i k} A_{j l}\right),
$$

i.e. (2.8). Now, using (2.7) and (2.8) we get immediately (2.9), which completes the proof of (i). (ii) is an obvious consequence of (i).

Lemma 2.2. Let $B$ be a symmetric $(0,2)$-tensor on a 2-dimensional semi-Riemannian manifold $(M, g)$. (i) [22, Lemma 2(iii)] The following identity is satisfied on $M$

$$
g \wedge B=\operatorname{tr}(B) G \text {. }
$$

(ii) The following identities are satisfied on $M$

$$
\begin{aligned}
B^{2} & =\operatorname{tr}(B) B+\frac{1}{2}\left(\operatorname{tr}\left(B^{2}\right)-(\operatorname{tr}(B))^{2}\right) g \\
Q\left(B, B^{2}\right) & =-\frac{1}{2}\left(\operatorname{tr}\left(B^{2}\right)-(\operatorname{tr}(B))^{2}\right) Q(g, B) .
\end{aligned}
$$

Proof. (ii) From (2.11) we get

$$
g_{h k} B_{i j}+g_{i j} B_{h k}-g_{h j} B_{i k}-g_{i k} B_{h j}=\operatorname{tr}(B)\left(g_{h k} g_{i j}-g_{h j} g_{i k}\right),
$$

where $B_{i j}$ and $B_{i j}^{2}$ are the local components of the tensors $B$ and $B^{2}$, respectively. Transvecting (2.13) with $B^{h k}=B_{i j} g^{h i} g^{k j}$ we obtain

$$
B_{i j}^{2}=\operatorname{tr}(B) B_{i j}+\frac{1}{2}\left(\operatorname{tr}\left(B^{2}\right)-(\operatorname{tr}(B))^{2}\right) g_{i j}
$$

i.e. (2.12). Further, we also have

$$
Q\left(B, B^{2}\right)=Q\left(B, \operatorname{tr}(B) B+\frac{1}{2}\left(\operatorname{tr}\left(B^{2}\right)-(\operatorname{tr}(B))^{2}\right) g\right)=-\frac{1}{2}\left(\operatorname{tr}\left(B^{2}\right)-(\operatorname{tr}(B))^{2}\right) Q(g, B),
$$

which completes the proof.

For symmetric $(0,2)$-tensors $E$ and $F$ we have

$$
Q(E, E \wedge F)=-\frac{1}{2} Q(F, E \wedge E), \quad E \wedge Q(E, F)=-\frac{1}{2} Q(F, E \wedge E)
$$


(see, e.g., [30, Section 3] and [27, eq. (3)]). In particular, from (2.14) we obtain

$$
Q(S, g \wedge S)=-\frac{1}{2} Q(g, S \wedge S), \quad Q(g, g \wedge S)=-Q(S, G) .
$$

Using now (1.5) and (2.15) we get

$$
Q(S, R)=Q(S, C)-\frac{1}{n-2} Q\left(g, \frac{1}{2} S \wedge S\right)-\frac{\kappa}{(n-2)(n-1)} Q(S, G) .
$$

We also have

$$
\begin{aligned}
& (g \wedge S) \cdot(g \wedge S)=-Q\left(S^{2}, G\right), G \cdot(g \wedge S)=Q(g, g \wedge S)=-Q(S, G), \\
& (g \wedge S) \cdot S=Q\left(g, S^{2}\right), G \cdot S=Q(g, S)
\end{aligned}
$$

(see, e.g., [28, Lemma 2.1 (ii)] and [69, Lemma 3.2]). Using (1.5) and (2.18) we obtain

$$
C \cdot S=R \cdot S-\frac{1}{n-2} Q\left(g, S^{2}\right)+\frac{\kappa}{(n-2)(n-1)} Q(g, S)
$$

(see, e.g., [45, p. 217]).

\section{Some CuRvature CONDitions}

A semi-Riemannian manifold $(M, g), n \geq 3$, is called semisymmetric if $R \cdot R=0$ on $M$ [85]. A semi-Riemannian manifold $(M, g), n \geq 3$, is said to be pseudosymmetric if the tensors $R \cdot R$ and $Q(g, R)$ are linearly dependent at every point of $M$ [22, 23, 24, 38]. This is equivalent on $\mathcal{U}_{R}=\left\{x \in M \mid R-\frac{\kappa}{(n-1) n} G \neq 0\right.$ at $\left.x\right\}$ to

$$
R \cdot R=L_{R} Q(g, R)
$$

where $L_{R}$ is some function on this set. We note that $\mathcal{U}_{S} \cup \mathcal{U}_{C}=\mathcal{U}_{R}$ (see, e.g. [28]). We mention that [38] is the first paper, in which manifolds satisfying (3.1) were called pseudosymmetric manifolds. It is easy to check that (3.1) is equivalent on $\mathcal{U}_{R}$ to $\left(R-L_{R} G\right) \cdot\left(R-L_{R} G\right)=0$. Evidently, every semisymmetric manifold is pseudosymmetric. The converse statement is not true. It seems that the Schwarzschild spacetime, the Kottler spacetime, the Reissner-Nordstrøm spacetime, as well as some Friedmann-Lemaître-Robertson-Walker spacetimes are the "oldest" examples of non-semisymmetric pseudosymmetric warped product manifolds (see, e.g., [39, 55]). Pseudosymmetric manifolds also are named Deszcz symmetric spaces (see, e.g., [88]). We also note that (3.1) implies

$$
R \cdot S=L_{R} Q(g, S), \quad R \cdot C=L_{R} Q(g, C) .
$$

The conditions (3.1) and (3.2) are equivalent on the set $\mathcal{U}_{S} \cap \mathcal{U}_{C}$ of any warped product manifold $M_{1} \times_{F} M_{2}$, with $\operatorname{dim} M_{1}=\operatorname{dim} M_{2}=2$ [22]. A semi-Riemannian manifold $(M, g), n \geq 3$, is called Ricci-pseudosymmetric if the tensors $R \cdot S$ and $Q(g, S)$ are linearly dependent at every point of $M[20,22,23,29,53]$. This is equivalent on $\mathcal{U}_{S}$ to

$$
R \cdot S=L_{S} Q(g, S)
$$

where $L_{S}$ is some function on this set. As it was mentioned in Introduction, every warped product manifold $\bar{M} \times_{F} \widetilde{N}$ of an 1-dimensional $(\bar{M}, \bar{g})$ manifold and an $(n-1)$-dimensional 
Einstein semi-Riemannian manifold $(\widetilde{N}, \widetilde{g}), n \geq 3$, with a warping function $F$, is a quasiEinstein manifold. Such warped products also are Ricci-pseudosymmetric manifolds, see, e.g., [12, Section 1] and Example 4.1 of this paper.

A semi-Riemannian manifold $(M, g), n \geq 4$, is said to be Weyl-pseudosymmetric if the tensors $R \cdot C$ and $Q(g, C)$ are linearly dependent at every point of $M$ [22, 23, 29]. This is equivalent on $\mathcal{U}_{C}$ to

$$
R \cdot C=L_{1} Q(g, C),
$$

where $L_{1}$ is some function on this set. Using (1.5), we can check that on every Einstein manifold $(M, g), n \geq 4$, (3.4) turns into $R \cdot R=L_{1} Q(g, R)$. For a presentation of results on the problem of the equivalence of pseudosymmetry, Ricci-pseudosymmetry and Weyl-pseudosymmetry we refer to [29, Section 4]. A semi-Riemannian manifold $(M, g), n \geq 4$, is said to have a pseudosymmetric Weyl conformal curvature tensor if the tensors $C \cdot C$ and $Q(g, C)$ are linearly dependent at every point of $M\left[15,22,23\right.$. This is equivalent on $\mathcal{U}_{C}$ to (1.9), where $L_{C}$ is some function on this set. We note that (1.9) is equivalent on $\mathcal{U}_{C}$ to $\left(C-L_{C} G\right) \cdot\left(C-L_{C} G\right)=0$.

As it was stated in [22], any warped product manifold $M_{1} \times_{F} M_{2}$, with $\operatorname{dim} M_{1}=\operatorname{dim} M_{2}=2$, satisfies (1.9). Thus in particular, the Schwarzschild spacetime, the Kottler spacetime and the Reissner-Nordstrøm spacetime satisfy (1.9). Recently manifolds with pseudosymmetric Weyl tensor were investigated in [28, 43]. Warped product manifolds $\bar{M} \times_{F} \widetilde{N}$, of dimension $\geq 4$, satisfying the condition (1.8) on $\mathcal{U}_{C} \subset \bar{M} \times_{F} \tilde{N}$, where $L$ is some function on this set, were studied in [14, 17]. In [17] necessary and sufficient conditions for $\bar{M} \times_{F} \widetilde{N}$ to be a manifold satisfying (1.8) are given. In particular, in that paper it was proved that any 4dimensional warped product manifold $\bar{M} \times_{F} \widetilde{N}$, with an 1-dimensional base $(\bar{M}, \bar{g})$, satisfies (1.8) [17, Theorem 4.1]. For details about the pseudosymmetric, Ricci-pseudosymmetric and Weyl-pseudosymmetric manifolds as well other conditions of this kind, named pseudosymmetry type curvature conditions, we refer to the papers: [12, 23, 29, 39, 64] and also references therein.

If $(M, g), n \geq 4$, is an Einstein semi-Riemannian manifold then $\mathcal{U}_{R}=\mathcal{U}_{C}$ and (1.5) yields

$$
C=R-\frac{\kappa}{(n-1) n} G \text {. }
$$

Theorem 3.1. If $(M, g), n \geq 4$, is a pseudosymmetric Einstein semi-Riemannian manifold satisfying (3.1) on $\mathcal{U}_{R} \subset M$ then on this set we have $R \cdot R-Q(S, R)=\left(L_{R}-\frac{\kappa}{n}\right) Q(g, C)$, $C \cdot C=\left(L_{R}-\frac{\kappa}{(n-1) n}\right) Q(g, C)$ and $C \cdot R+R \cdot C=Q(S, C)+\left(2 L_{R}-\frac{\kappa}{n-1}\right) Q(g, C)$.

Proof. The second condition of our assertion was proved in [15, Theorem 3.1]. Further, using (3.1) and (3.5) we obtain $R \cdot C=L_{R} Q(g, C)$ and

$$
\begin{aligned}
& R \cdot R-Q(S, R)=\left(L_{R}-\frac{\kappa}{n}\right) Q\left(g, R-\frac{\kappa}{(n-1) n} G\right)=\left(L_{R}-\frac{\kappa}{n}\right) Q(g, C), \\
& C \cdot R+R \cdot C=\left(R-\frac{\kappa}{(n-1) n} G\right) \cdot R+L_{R} Q(g, C) \\
& =R \cdot R-\frac{\kappa}{(n-1) n} G \cdot R+L_{R} Q(g, C)=\left(L_{R}-\frac{\kappa}{(n-1) n}\right) Q(g, R)+L_{R} Q(g, C) \\
& =\left(2 L_{R}-\frac{\kappa}{(n-1) n}\right) Q(g, C)=Q(S, C)+\left(2 L_{R}-\frac{\kappa}{n-1}\right) Q(g, C),
\end{aligned}
$$


completing the proof.

In [86, Section 2] a class of 4-dimensional Einstein Riemannian manifolds was defined and investigated. As it was stated in [13, Remark 5.1] those manifolds are pseudosymmetric. If a non-quasi-Einstein semi-Riemannian manifold $(M, g), n \geq 4$, satisfies on $\mathcal{U}_{S} \cap \mathcal{U}_{C} \subset M$ (3.1) and (1.8) or (3.1) and (1.9), then (1.7) holds on this set ([58, Theorem 3.2 (ii)], [41, Lemma 4.1]). We also have the following converse statement.

Theorem 3.2. [29, 60] If $(M, g), n \geq 4$, is a semi-Riemannian manifold satisfying (1.7) on $\mathcal{U}_{S} \cap \mathcal{U}_{C} \subset M$ then on this set we have

$$
\begin{aligned}
& S^{2}=\alpha_{1} S+\alpha_{2} g, \quad \alpha_{1}=\kappa+\frac{(n-2) \mu-1}{\phi}, \alpha_{2}=\frac{\mu \kappa+(n-1) \eta}{\phi}, \\
& R \cdot C=L_{R} Q(g, C), \quad L_{R}=\frac{1}{\phi}\left((n-2)\left(\mu^{2}-\phi \eta\right)-\mu\right), \\
& R \cdot R=L_{R} Q(g, R), \quad R \cdot S=L_{R} Q(g, S), \\
& R \cdot R=Q(S, R)+L Q(g, C), \quad L=L_{R}+\frac{\mu}{\phi}=\frac{n-2}{\phi}\left(\mu^{2}-\phi \eta\right), \\
& C \cdot C=L_{C} Q(g, C), \quad L_{C}=L_{R}+\frac{1}{n-2}\left(\frac{\kappa}{n-1}-\alpha_{1}\right), \\
& C \cdot R=L_{C} Q(g, R), C \cdot S=L_{C} Q(g, S), \\
& R \cdot C-C \cdot R=\frac{1}{n-2} Q(S, R)+\left(\frac{(n-1) \mu-1}{(n-2) \phi}+\frac{\kappa}{n-1}\right) Q(g, R) \\
& R \cdot C-C \cdot R=\left(\frac{1}{\phi}\left(\mu-\frac{\mu((n-1) \mu-1)-(n-1) \phi \eta}{n-2}\right)+\frac{\kappa}{n-1}\right) Q(S, G), \\
&+\frac{(n-2) \phi}{n-1}\left(\frac{\mu}{\phi}\left(\mu-\frac{1}{n-2}\right)-\eta\right) Q(S, G) .
\end{aligned}
$$

Remark 3.1. Let the curvature tensor $R$ of a semi-Riemannian manifold $(M, g), n \geq 4$, has the decomposition (1.7) on $\mathcal{U}_{S} \cap \mathcal{U}_{C} \subset M$. In [42, Lemma 3.2] it was shown that the decomposition (1.7) is unique on this set.

Proposition 3.3. If $(M, g), n \geq 4$, is a semi-Riemannian manifold satisfying (1.7) on $\mathcal{U}_{S} \cap$ $\mathcal{U}_{C} \subset M$ then (1.10) and (1.11) hold on this set.

Proof. On $\mathcal{U}_{S} \cap \mathcal{U}_{C} \subset M$ we have $C \cdot R=Q(S, C)+\left(L_{R}-\frac{\kappa}{n-1}\right) Q(g, C)$ [69, eq. (37)], where the function $L_{R}$ is defined by (1.10) (see also Theorem 3.1). But this, together with $R \cdot C=L_{R} Q(g, C)$ and $L+L_{C}-\frac{1}{(n-2) \phi}=2 L_{R}-\frac{\kappa}{n-1}$ (see Theorem 3.1), completes the proof.

Theorem 3.4. Let $(M, g), n \geq 4$, be a semi-Riemannian manifold. (i) The identity (1.13) is satisfied on $M$. (ii) If (1.8), with some function $L$, is satisfied on $\mathcal{U}_{C} \subset M$ then (1.14) holds on this set. (iii) If (1.8) and (1.9), with some functions $L$ and $L_{C}$, are satisfied on $\mathcal{U}_{C} \subset M$ then (1.15) holds on this set. (iv) If $(M, g)$ is a non-Einstein and non-conformally flat semiRiemannian manifold satisfying on $\mathcal{U}_{S} \cap \mathcal{U}_{C} \subset M$ the conditions: (1.1), and (1.8) and (1.9), 
with some functions $L$ and $L_{C}$, then (1.16) holds on this set. (v) The equation (1.17) is satisfied on the Gödel spacetime.

Proof. (i) We have (cf. [77, Section 1])

$$
(n-2)^{2}(C-R) \cdot(C-R)=\left(g \wedge S-\frac{\kappa}{n-1} G\right) \cdot\left(g \wedge S-\frac{\kappa}{n-1} G\right),
$$

which yields

$$
(n-2)^{2}(C \cdot C-R \cdot C-C \cdot R+R \cdot R)=(g \wedge S) \cdot(g \wedge S)-\frac{\kappa}{n-1} G \cdot(g \wedge S) .
$$

But this, by (2.17), turns into (1.13). (ii) It is easy to see that (1.13), by making use of (1.9) and the identities (2.15) and (2.16) turns into (1.14). (iii) Relations (2.16), (1.9), (1.8) and (1.13) yield

$$
\begin{aligned}
& C \cdot R+R \cdot C=Q(S, R)+\left(L+L_{C}\right) Q(g, C)+\frac{1}{(n-2)^{2}} Q\left(S^{2}-\frac{\kappa}{n-1} S, G\right) \\
= & Q(S, C)+\left(L+L_{C}\right) Q(g, C)-\frac{1}{n-2} Q\left(g, \frac{1}{2} S \wedge S\right)+\frac{1}{(n-2)^{2}} Q\left(S^{2}-\kappa S, G\right),
\end{aligned}
$$

which by (2.14) turns into (1.15). (iii) It is easy to see that the conditions (1.1), (1.3), (1.4), (1.15) and $Q(g, G)=0$ lead to (1.16). (iv-v) The Ricci tensor $S$ of the Gödel spacetime $(M, g)$ satisfies $S=\kappa \omega \otimes \omega$, where $\omega$ is an 1-form [62]. From the last equation we get easily $S \wedge S=0$ and $S^{2}=\kappa S$. It is also known that $R \cdot R=Q(S, R)$ and $C \cdot C=\frac{\kappa}{6} Q(g, C)$ hold on $M$ [43, Theorem 2]. Now (1.16) yields (1.17). Our theorem is thus proved.

Remark 3.2. In [43, Section 4(v)] it was shown that on the Gödel spacetime the tensors $R \cdot C$, $C \cdot R, Q(g, R), Q(S, R), Q(g, C)$ and $Q(S, C)$ are linearly dependent.

We also have the following result.

Proposition 3.5. cf. [28, Proposition 3.2, Theorem 3.3, Theorem 4.4] If $(M, g), n \geq 4$, is a semi-Riemannian manifold satisfying on $\mathcal{U}_{S} \cap \mathcal{U}_{C} \subset M$ the conditions (1.8), (1.9) and

$$
R \cdot S=Q(g, D),
$$

where $D$ is a symmetric (0,2)-tensor, then (3.1) holds on this set. Moreover, at every point of $\mathcal{U}_{S} \cap \mathcal{U}_{C}$ we have rank $\left(S-\alpha_{1} g\right)=1$ or rank $\left(S-\alpha_{1} g\right) \geq 2$ and $(1.7)$, where $\alpha_{1}=\frac{1}{2}\left(\frac{\kappa}{n-1}-L+L_{C}\right)$.

The last proposition, together with Proposition 3.3 and Theorem 3.4(iv), yields

Corollary 3.6. If $(M, g), n \geq 4$, is a semi-Riemannian manifold satisfying on $\mathcal{U}_{S} \cap \mathcal{U}_{C} \subset M$ the conditions (1.8), (1.9) and (3.6) then $C \cdot R+R \cdot C=Q(S, C)+L_{2} Q(g, C)$ holds on $\mathcal{U}_{S} \cap \mathcal{U}_{C}$, where $L_{2}$ is some function on this set.

Let $M, n=\operatorname{dim} M \geq 4$, be a connected hypersurface isometrically immersed in a semiRiemannian space of constant curvature $N_{s}^{n+1}(c)$, with signature $(s, n+1-s)$, where $c=\frac{\widetilde{\kappa}}{n(n+1)}$ and $\widetilde{\kappa}$ is its scalar curvature. It is known that (1.8) holds on $M$. Precisely,

$$
R \cdot R=Q(S, R)-\frac{(n-2) \widetilde{\kappa}}{n(n+1)} Q(g, C)
$$

on $M$ [54, Proposition 3.1]. Now, as an immediate consequence of Theorem 3.3, we have 
Theorem 3.7. Let $M$ is a hypersurface isometrically immersed in $N_{s}^{n+1}(c), n \geq 4$. Then

$$
\begin{aligned}
C \cdot R+R \cdot C= & Q(S, C)-\frac{(n-2) \widetilde{\kappa}}{n(n+1)} Q(g, C)+C \cdot C \\
& -\frac{1}{(n-2)^{2}} Q\left(g, \frac{n-2}{2} S \wedge S-\kappa g \wedge S+g \wedge S^{2}\right)
\end{aligned}
$$

holds on $M$. Moreover, if (1.9) is satisfied on $\mathcal{U}_{S} \cap \mathcal{U}_{C} \subset M$ then on this set we have

$$
\begin{aligned}
C \cdot R+R \cdot C= & Q(S, C)+\left(L_{C}-\frac{(n-2) \widetilde{\kappa}}{n(n+1)}\right) Q(g, C) \\
& -\frac{1}{(n-2)^{2}} Q\left(g, \frac{n-2}{2} S \wedge S-\kappa g \wedge S+g \wedge S^{2}\right) .
\end{aligned}
$$

If $M$ is a quasi-Einstein hypersurface satisfying (1.1) and (1.8) on $\mathcal{U}_{S} \cap \mathcal{U}_{C}$ then on this set we have

$$
C \cdot R+R \cdot C=Q(S, C)+\left(L_{C}-\frac{(n-2) \widetilde{\kappa}}{n(n+1)}\right) Q(g, C) .
$$

It is known that every 2-quasi-umbilical hypersurface in a semi-Riemannian space of constant curvature $N_{s}^{n+1}(c), n \geq 4$, satisfies (1.9) [57, Theorem 3.1]. Now Theorem 3.4 yields

Theorem 3.8. If $M$ is a 2-quasi-umbilical hypersurface isometrically immersed in $N_{s}^{n+1}(c)$, $n \geq 4$, then (3.9) holds on $\mathcal{U}_{S} \cap \mathcal{U}_{C} \subset M$.

Let $M$ be an $n$-dimensional Chen ideal submanifold of codimension $m$ isometrically immersed in an Euclidean space $\mathbb{E}^{n+m}, n \geq 4, m \geq 1$ [9, 10. It is known that (1.8) and (1.9) hold on $\mathcal{U}_{C} \subset M$ ([49, Theorem 1], see also [11, Section 6] and [50, Section 3.1]). Now Theorem 3.3(ii) yields

Theorem 3.9. If $M, n \geq 4$, is a Chen ideal submanifold of codimension $m, m \geq 1$, isometrically immersed in an Euclidean space $\mathbb{E}^{n+m}$ then (1.15) holds on this set.

Remark 3.3. (i) We refer to [61] for further results on quasi-Einstein hypersurfaces $M$ in $N_{s}^{n+1}(c), n \geq 4$, satisfying (1.9). (ii) We refer to [50] for curvature properties of pseudosymmetry type of Chen ideal submanifolds in an Euclidean space. (iii) From (3.7) it follows that every Einstein hypersurface $M$ in $N_{s}^{n+1}(c), n \geq 4$, is a pseudosymmetric manifold satisfying (3.1) and $L_{R}=\frac{\kappa}{n}-\frac{(n-2) \widetilde{\kappa}}{n(n+1)}$ on $\mathcal{U}_{R} \subset M$ (cf. [23, Section 5.5]). Now from Theorem 3.1 we have

$$
R \cdot C+C \cdot R=Q(S, C)+\frac{n-2}{n}\left(\frac{\kappa}{n-1}-\frac{2 \widetilde{\kappa}}{n+1}\right) Q(g, C)
$$

on $\mathcal{U}_{R}$. We refer to [75] for examples of semisymmetric Einstein hypersurfaces in some semiRiemannian spaces of constant curvature. (iv) Let $M$ be a hypersurface in $N_{s}^{n+1}(c), n \geq 4$. If at every point of $\mathcal{U}_{C} \subset M$ the tensor $H^{2}$, the square of the second fundamental tensor $H$ of $M$, is a linear combination of $H$ and the metric tensor $g$ of $M$ then (1.9) holds on $\mathcal{U}_{C}$ (see, e.g., [57, Section 1]). Moreover, in view of Theorem 3.5(iii), (1.15) is satisfied on $\mathcal{U}_{C}$. 


\section{WARPED PRODUCT MANIFOLDS}

Let now $(\bar{M}, \bar{g})$ and $(\widetilde{N}, \widetilde{g}), \operatorname{dim} \bar{M}=p, \operatorname{dim} N=n-p, 1 \leq p<n$, be semi-Riemannian manifolds covered by systems of charts $\left\{U ; x^{a}\right\}$ and $\left\{V ; y^{\alpha}\right\}$, respectively. Let $F$ be a positive smooth function on $\bar{M}$. The warped product $\bar{M} \times_{F} N$ of $(\bar{M}, \bar{g})$ and $(\widetilde{N}, \widetilde{g})$ is the product manifold $\bar{M} \times \widetilde{N}$ with the metric $g=\bar{g} \times_{F} \widetilde{g}=\pi_{1}^{*} \bar{g}+\left(F \circ \pi_{1}\right) \pi_{2}^{*} \widetilde{g}$, where $\pi_{1}: \bar{M} \times \widetilde{N} \longrightarrow \bar{M}$ and $\pi_{2}: \bar{M} \times \widetilde{N} \longrightarrow \widetilde{N}$ are the natural projections on $\bar{M}$ and $\widetilde{N}$, respectively [6, 71, 76]. Let $\left\{U \times V ; x^{1}, \ldots, x^{p}, x^{p+1}=y^{1}, \ldots, x^{n}=y^{n-p}\right\}$ be a product chart for $\bar{M} \times \widetilde{N}$. The local components $g_{i j}$ of the metric $g=\bar{g} \times_{F} \widetilde{g}$ with respect to this chart are the following $g_{i j}=\bar{g}_{a b}$ if $i=a$ and $j=b, g_{i j}=F \widetilde{g}_{\alpha \beta}$ if $i=\alpha$ and $j=\beta$, and $g_{i j}=0$ otherwise, where $a, b, c, d, f \in$ $\{1, \ldots, p\}, \alpha, \beta, \gamma, \delta \in\{p+1, \ldots, n\}$ and $h, i, j, k, l, m, r, s \in\{1,2, \ldots, n\}$. We will denote by bars (resp., by tildes) tensors formed from $\bar{g}$ (resp., $\widetilde{g}$ ). The local components

$$
\Gamma_{i j}^{h}=\frac{1}{2} g^{h s}\left(\partial_{i} g_{j s}+\partial_{j} g_{i s}-\partial_{s} g_{i j}\right), \quad \partial_{j}=\frac{\partial}{\partial x^{j}},
$$

of the Levi-Civita connection $\nabla$ of $\bar{M} \times_{F} \widetilde{N}$ are the following

$\Gamma_{b c}^{a}=\bar{\Gamma}_{b c}^{a}, \quad \Gamma_{\beta \gamma}^{\alpha}=\widetilde{\Gamma}_{\beta \gamma}^{\alpha}, \quad \Gamma_{\alpha \beta}^{a}=-\frac{1}{2} \bar{g}^{a b} F_{b} \widetilde{g}_{\alpha \beta}, \quad \Gamma_{a \beta}^{\alpha}=\frac{1}{2 F} F_{a} \delta_{\beta}^{\alpha}, \quad \Gamma_{\alpha b}^{a}=\Gamma_{a b}^{\alpha}=0, \quad F_{a}=\frac{\partial F}{\partial x^{a}}$ (see, e.g., [48, 72]). The local components

$$
R_{h i j k}=g_{h s} R_{i j k}^{s}=g_{h s}\left(\partial_{k} \Gamma_{i j}^{s}-\partial_{j} \Gamma_{i k}^{s}+\Gamma_{i j}^{r} \Gamma_{r k}^{s}-\Gamma_{i k}^{r} \Gamma_{r j}^{s}\right),
$$

of the Riemann-Christoffel curvature tensor $R$ and the local components $S_{i j}$ of the Ricci tensor $S$ of the warped product $\bar{M} \times_{F} \widetilde{N}$ which may not vanish identically are the following:

$$
\begin{aligned}
R_{a b c d} & =\bar{R}_{a b c d}, \quad R_{\alpha a b \delta}=-\frac{1}{2} T_{a b} \widetilde{g}_{\alpha \delta}, \quad R_{\alpha \beta \gamma \delta}=F \widetilde{R}_{\alpha \beta \gamma \beta}-\frac{1}{4} \Delta_{1} F \widetilde{G}_{\alpha \beta \gamma \delta}, \\
S_{a b} & =\bar{S}_{a b}-\frac{n-p}{2} \frac{1}{F} T_{a b}, \quad S_{\alpha \beta}=\tilde{S}_{\alpha \beta}-\frac{1}{2}\left(\operatorname{tr}(T)+\frac{n-p-1}{2 F} \Delta_{1} F\right) \widetilde{g}_{\alpha \beta}, \\
T_{a b} & =\bar{\nabla}_{a} F_{b}-\frac{1}{2 F} F_{a} F_{b}, \quad \operatorname{tr}(T)=\bar{g}^{a b} T_{a b}, \quad \Delta_{1} F=\Delta_{1 \bar{g}} F=\bar{g}^{a b} F_{a} F_{b},
\end{aligned}
$$

where $T$ is the $(0,2)$-tensor with the local components $T_{a b}$. The scalar curvature $\kappa$ of $\bar{M} \times_{F} \widetilde{N}$ satisfies the following relation

$$
\kappa=\bar{\kappa}+\frac{1}{F} \widetilde{\kappa}-\frac{n-p}{F}\left(\operatorname{tr}(T)+\frac{n-p-1}{4 F} \Delta_{1} F\right) .
$$

Warped products play an important role in Riemannian geometry (see, e.g., [5, 6, 72, 76]) as well as in the general relativity theory (see, e.g., [7, 63, 76, 84]). Many well-known spacetimes of this theory, i.e. solutions of the Einstein field equations, are warped products, e.g. the Schwarzschild, Kottler, Reissner-Nordstrøm, Reissner-Nordstrøm-de Sitter, Vaidya, VaidyaKottler, Vaidya-Reissner-Nordstrøm, Vaidya-Bonnor, as well as Robertson-Walker spacetimes. We recall that a warped product $\bar{M} \times_{F} \widetilde{N}$ of an 1-dimensional manifold $(\bar{M}, \bar{g}), \bar{g}_{11}=-1$, and a 3-dimensional Riemannian space of constant curvature $(\widetilde{N}, \widetilde{g})$, with a warping function $F$, is said to be a Robertson-Walker spacetime (see, e.g., [63, 76, 84]). It is well-known that the Robertson-Walker spacetimes are conformally flat quasi-Einstein manifolds. More generally, one also considers warped products $\bar{M} \times_{F} \widetilde{N}$ of $(\bar{M}, \bar{g}), \operatorname{dim} \bar{M}=1, \bar{g}_{11}=-1$, 
with a warping function $F$ and an $(n-1)$-dimensional Riemannian manifold $(\widetilde{N}, \widetilde{g}), n \geq 4$. Such warped products are called generalized Robertson-Walker spacetimes [1, 2]. We mention that Einstein generalized Robertson-Walker spacetimes were classified in [2]. Curvature conditions of pseudosymmetry type on spacetimes have been considered among others in [3, 12, 13, 15, 16, 40, 43, 48, 51, 55, 68, 69].

Example 4.1. The warped product manifold $\bar{M} \times_{F} \widetilde{N}$, of an 1-dimensional manifold $(\bar{M}, \bar{g})$, $\bar{g}_{11}= \pm 1$, and an $(n-1)$-dimensional semi-Riemannian Einstein manifold $(\tilde{N}, \widetilde{g}), n \geq 5$, which is not of constant curvature, with a warping function $F$, satisfies on $\mathcal{U}_{S} \cap \mathcal{U}_{C} \subset \bar{M} \times_{F} \widetilde{N}$ :

$$
\begin{aligned}
& R \cdot S=L_{S} Q(g, S), \quad L_{S}=-\frac{\operatorname{tr} T}{2 F}, \operatorname{rank}(S-\alpha g)=1, \alpha=\frac{\kappa}{n-1}-L_{S}, \\
& (n-2)(R \cdot C-C \cdot R)=Q(S, R)-L_{S} Q(g, R),
\end{aligned}
$$

[12, Theorem 4.1]. Furthermore, using (1.3), (1.5), (2.15), (2.16) and (4.5) we get

$$
\begin{aligned}
Q(g, R) & =Q(g, C)-\frac{1}{n-2} Q(S, G), \\
Q(S, R) & =Q(S, C)+\frac{1}{n-2}\left(\alpha-\frac{\kappa}{n-1}\right) Q(S, G), \\
(n-2)(R \cdot C-C \cdot R) & =Q(S, C)-L_{S} Q(g, C) .
\end{aligned}
$$

Using Theorem 3.4(i)-(iii), [17, Theorem 4.1] and [56, Theorem 2] we obtain

Theorem 4.1. Let $\bar{M} \times_{F} \widetilde{N}$ be the warped product manifold of an 1-dimensional manifold $(\bar{M}, \bar{g}), \bar{g}_{11}= \pm 1$, and a 3-dimensional semi-Riemannian manifold $(\tilde{N}, \widetilde{g})$. If $(\tilde{N}, \widetilde{g})$ is not a space of constant curvature then (1.8) and (1.14) hold on $\mathcal{U}_{C} \subset \bar{M} \times_{F} \widetilde{N}$. Moreover, if $(\tilde{N}, \widetilde{g})$ is a quasi-Einstein manifold then (1.9) and (1.15) hold on $\mathcal{U}_{S} \cap \mathcal{U}_{C} \subset \bar{M} \times_{F} \tilde{N}$.

The Ricci tensor of the following 3-dimensional Riemannian manifolds $(\tilde{N}, \widetilde{g})$ : the Berger spheres, the Heisenberg group $N i l_{3}, \widetilde{P S(2, \mathbb{R})}$ - the universal covering of the Lie group $P S L(2, \mathbb{R})$ and the Lie group $S_{3}[74$, Section 3], a Riemannian manifold isometric to an open part of the Cartan hypersurface [27, Section 2] and some three-spheres of Kaluza-Klein type [8, Theorem $2(i i)_{a}$ ] have exactly two distinct eigenvalues. Evidently, these manifolds are quasi-Einstein, and in a consequence, pseudosymmetric (see, e.g., [56, Theorem 1]). For further examples of 3-dimensional quasi-Einstein manifolds we refer to [4] (Thurston geometries and warped product manifolds) and [70] (manifolds with constant Ricci principal curvatures).

Theorem 4.1 leads to the following result.

Theorem 4.2. The conditions (1.8), (1.9) and (1.15) are satisfied on the warped product manifold $\bar{M} \times_{F} \widetilde{N}$ of an 1-dimensional manifold $(\bar{M}, \bar{g}), \bar{g}_{11}= \pm 1$, and the 3-dimensional Riemannian manifold $(\widetilde{N}, \widetilde{g})$ such as: the Berger sphere, $\left.N_{i l}, \widetilde{P L(2,} \mathbb{R}\right), S_{3} l_{3}$, a Riemannian manifold isometric to an open part of the Cartan hypersurface, or some three-spheres of KaluzaKlein type.

Using [17, Theorem 4.2], [21, Theorem 3.5] and [56, Theorem 3] we can prove 
Theorem 4.3. If $\bar{M} \times_{F} \widetilde{N}$ is the warped product manifold of an 1-dimensional manifold $(\bar{M}, \bar{g})$, $\bar{g}_{11}= \pm 1$, and an $(n-1)$-dimensional quasi-Einstein conformally flat semi-Riemannian manifold $(\widetilde{N}, \widetilde{g}), n \geq 5$, then the conditions (1.8), (1.9) and (1.15) are satisfied on $\mathcal{U}_{C} \subset \bar{M} \times_{F} \tilde{N}$.

We mention that recently curvature conditions of pseudosymmetry type of four-dimensional Thurston geometries were investigated in 67].

\section{Pseudosymmetric WARPed PRODUCT MANIFOldS}

In this section we present some results on pseudosymmetric warped product manifolds.

Theorem 5.1. 24] The Riemann-Christoffel curvature tensor $R$ of the warped product manifold $\bar{M} \times_{F} \tilde{N}$, with $\operatorname{dim} \bar{M}=p, \operatorname{dim} \tilde{N}=n-p, 1 \leq p \leq n-1, n \geq 3$, satisfies (3.1), i.e. $R \cdot R=L_{R} Q(g, R)$, on some coordinate domain of a point $x \in \mathcal{U}_{R} \subset \bar{M} \times_{F} \tilde{N}$ if and only if the following relations are satisfied on this set

$$
\begin{aligned}
(\bar{R} \cdot \bar{R})_{a b c d e f} & =L_{R} Q(\bar{g}, \bar{R})_{a b c d e f}, \\
2 H_{d}^{f} \bar{R}_{f a b c} & =\frac{1}{F}\left(T_{a c} H_{b d}-T_{a b} H_{c d}\right), \\
H_{a d}\left(\widetilde{R}_{\delta \alpha \beta \gamma}-\frac{\Delta_{1} F}{4 F} \widetilde{G}_{\delta \alpha \beta \gamma}\right) & =-\frac{1}{2} T_{d}^{f} H_{f a} \widetilde{G}_{\delta \alpha \beta \gamma}, \\
(\widetilde{R} \cdot \widetilde{R})_{\alpha \beta \gamma \delta \lambda \mu} & =\left(F L_{R}+\frac{\Delta_{1} F}{4 F}\right) Q(\widetilde{g}, \widetilde{R})_{\alpha \beta \gamma \delta \lambda \mu},
\end{aligned}
$$

where $T_{a b}$ is defined by (4.3) and

$$
H_{a b}=\frac{1}{2} T_{a b}+F L_{R} \bar{g}_{a b} .
$$

Proposition 5.2. Let $\bar{M} \times_{F} \widetilde{N}$ be the warped product of semi-Riemannian manifolds $(\bar{M}, \bar{g})$ and $(\widetilde{N}, \widetilde{g}), \operatorname{dim} \bar{M}=p, \operatorname{dim} \widetilde{N}=n-p, 2 \leq p \leq n-1, n \geq 4$, with the warping function $F$, and let $(\bar{M}, \bar{g})$ and $(\widetilde{N}, \widetilde{g})$ be a spaces of constant curvature, provided that $p \geq 3$ and $n-p \geq 3$, respectively. (i) cf. [24, Corollary 2.1] The warped product $\bar{M} \times_{F} \tilde{N}$ satisfies $R \cdot R=L_{R} Q(g, R)$, i.e. (3.1), on some coordinate domain of a point $x \in \mathcal{U}_{R} \subset \bar{M} \times_{F} \tilde{N}$ if and only if the following two relations are satisfied on this set

$$
\begin{aligned}
H_{a c} H_{b d}-H_{a b} H_{c d} & =F\left(\frac{\bar{\kappa}}{(p-1) p}-L_{R}\right)\left(\bar{g}_{a b} H_{c d}-\bar{g}_{a c} H_{b d}\right), \\
\widetilde{\kappa} H_{a d} & =(n-p)(n-p-1)\left(\left(F L_{R}+\frac{\Delta_{1} F}{4 F}\right) H_{a d}-H_{a d}^{2}\right),
\end{aligned}
$$

where $H_{a d}^{2}=\bar{g}^{\text {ef }} H_{a e} H_{d f}$ and $\bar{\kappa}$ and $\widetilde{\kappa}$ are the scalar curvatures of $(\bar{M}, \bar{g})$ and $(\tilde{N}, \widetilde{g})$, respectively. Moreover, if $n-p \geq 2$ then (5.3) is equivalent to

$$
H_{a d}^{2}=\left(\frac{F \bar{\kappa}}{(p-1) p}-\frac{\widetilde{\kappa}}{(n-p-1)(n-p)}+\frac{\Delta_{1} F}{4 F}\right) H_{a d} .
$$

(ii) If $H=\frac{\operatorname{tr} H}{p} \bar{g}$ is satisfied on some coordinate domain $U$ of a point $x \in \mathcal{U}_{R} \subset \bar{M} \times_{F} \widetilde{N}$, for certain function $L_{R}$, then $T=\frac{\operatorname{tr} T}{p} \bar{g}$ on $U$. Moreover, $H=0$ and (3.1) hold on $U$, provided 
that $L_{R}=-\frac{\operatorname{tr} T}{2 p F}$. (iii) Let $\bar{M} \times_{F} \tilde{N}$ be the warped product satisfying $R \cdot R=L_{R} Q(g, R)$. If $H \neq \frac{\operatorname{tr} H}{p} \bar{g}$ at a point $x \in \mathcal{U}_{R} \subset \bar{M} \times_{F} \widetilde{N}$ then on some neighbourhood $U \subset \mathcal{U}_{R}$ of $x$ we have

$$
\text { (a) } \quad L_{R}=\frac{\bar{\kappa}}{(p-1) p}, \quad \text { (b) } \operatorname{rank}(H)=1 .
$$

Proof. (i) This assertion is a consequence of Theorem 5.1 and the definition of $H_{a b}$ (see (5.1)). (ii) From our assumption, by (5.1), it follows that $T$ is proportional to $g$. It is obvious that if we set $L_{R}=-\frac{\operatorname{tr} T}{2 p F}$ then (5.1) gives $H=0$. Now (i) completes the proof of (ii). (iii) From (5.2) we have

$$
H_{c d} H_{a b}-H_{a c} H_{b d}=F\left(\frac{\bar{\kappa}}{(p-1) p}-L_{R}\right)\left(\bar{g}_{b d} H_{a c}-\bar{g}_{c d} H_{a b}\right),
$$

This, together with (5.2), yields

$$
\left(\frac{\bar{\kappa}}{(p-1) p}-L_{R}\right)\left(\bar{g}_{a b} H_{c d}-\bar{g}_{a c} H_{b d}+\bar{g}_{b d} H_{a c}-\bar{g}_{c d} H_{a b}\right)=0,
$$

which by contraction with $g^{a b}$ gives $\left(\frac{\bar{\kappa}}{(p-1) p}-L_{R}\right)\left(H_{c d}-\frac{\operatorname{tr} H}{p} \bar{g}_{c d}\right)=0$. From this, by our assumption, we get immediately (5.5) (a). Now (5.2), by (5.5) (a), reduces to

$$
H_{a c} H_{b d}-H_{a b} H_{c d}=0,
$$

which is equivalent to (5.5) (b). Our proposition is thus proved.

Let $\bar{M} \times_{F} \widetilde{N}$ be the warped product of semi-Riemannian manifolds $(\bar{M}, \bar{g}), \operatorname{dim} \bar{M}=p$, and $(\widetilde{N}, \widetilde{g}), \operatorname{dim} \widetilde{N}=n-p, 2 \leq p \leq n-2$, with the warping function $F$, and let $(\bar{M}, \bar{g})$ and $(\widetilde{N}, \widetilde{g})$ be spaces of constant curvature, provided that $p \geq 3$ and $n-p \geq 3$, respectively, satisfying (3.1) on $\mathcal{U}_{R} \subset \bar{M} \times_{F} \widetilde{N}$. Moreover, let $H \neq \frac{\operatorname{tr} H}{p} \bar{g}$ at a point $x \in \mathcal{U}_{R}$. We note that from (4.2) it follows that $S \neq \frac{\kappa}{n} g$ at this point. Further, in view of Proposition 5.2, (5.4), (5.5) and (5.6) hold on some neighbourhood $U \subset \mathcal{U}_{R}$ of $x$. From (5.6), by a suitable contraction, it follows that $H^{2}=\operatorname{tr} H H$ on $U$. The last equation and (5.4) yield

$$
\frac{\bar{\kappa}}{p}+\frac{\widetilde{\kappa}}{(n-p-1)(n-p) F}+\frac{\operatorname{tr} T}{2 F}-\frac{\Delta_{1} F}{4 F^{2}}=0 .
$$

We note that if $p=2$ then (7.2), (7.4), (17.5) and (5.7) lead to $\rho_{0}=\rho=0$, and $C=0$.

From the above presented considerations it follows

Theorem 5.3. Let $\bar{M} \times_{F} \widetilde{N}$ be the warped product of a 2-dimensional semi-Riemannian manifold $(\bar{M}, \bar{g})$ and an $(n-2)$-dimensional semi-Riemannian manifold $(\widetilde{N}, \widetilde{g}), n \geq 4$, with the warping function $F$, and let $(\tilde{N}, \widetilde{g})$ be a space of constant curvature, provided that $n \geq 5$. The manifold $\bar{M} \times_{F} \widetilde{N}$ satisfies (3.1), i.e. $R \cdot R=L_{R} Q(g, R)$, on some coordinate domain $U$ of a point $x \in \mathcal{U}_{S} \cap \mathcal{U}_{C} \subset \bar{M} \times_{F} \widetilde{N}$ if and only if on $U$ we have

$$
H=\frac{1}{2} T+F L_{R} \bar{g}=\frac{1}{2} T+F\left(-\frac{\operatorname{tr} T}{4 F}\right) \bar{g}=\frac{1}{2}\left(T-\frac{\operatorname{tr} T}{2} \bar{g}\right)=0,
$$

i.e. $T_{a b}$ is proportional to $\bar{g}_{a b}$ on $U$. 
At the end of this section we recall the following result of [47].

Theorem 5.4. cf. [47, Theorem 4.1] Let $\bar{M} \times_{F} \tilde{N}$ be the warped product of a 2-dimensional semi-Riemannian manifold $(\bar{M}, \bar{g})$ and an $(n-2)$-dimensional semi-Riemannian manifold $(\tilde{N}, \widetilde{g})$, $n \geq 4$, with the warping function $F$, and let $(\widetilde{N}, \widetilde{g})$ be a space of constant curvature, provided that $n \geq 5$. If the tensor $T_{a b}$, defined by (4.3), is proportional to $\bar{g}_{a b}$ at every point of $\mathcal{U}_{S} \cap \mathcal{U}_{C} \subset \bar{M} \times_{F} \tilde{N}$ then (1.7) holds on this set.

\section{WARPED PRODUCT MANIFOLDS With 2-DIMENSIONAL BASE AND EINSTEINIAN FIBRE}

Let $\bar{M} \times_{F} \tilde{N}$ be the warped product manifold of a 2-dimensional manifold $(\bar{M}, \bar{g})$ and an $(n-2)$-dimensional semi-Riemannian manifold $(\widetilde{N}, \widetilde{g}), n \geq 4$, with a warping function $F$, and let $(\widetilde{N}, \widetilde{g})$ be an Einstein manifold, provided that $n \geq 5$. Now (4.2) turns into

$$
\begin{aligned}
S_{a d} & =\frac{\bar{\kappa}}{2} g_{a b}-\frac{n-2}{2 F} T_{a b}, \quad S_{\alpha \beta}=\tau_{1} g_{\alpha \beta}, \\
\tau_{1} & =\frac{\widetilde{\kappa}}{(n-2) F}-\frac{\operatorname{tr}(T)}{2 F}-(n-3) \frac{\Delta_{1} F}{4 F^{2}} .
\end{aligned}
$$

From (6.1) it follows that $T_{a b}$ is proportional to $\bar{g}_{a b}$ at a point of $\mathcal{U}_{S} \cap \mathcal{U}_{C} \subset \bar{M} \times_{F} \tilde{N}$ if and only if $S_{a b}$ is proportional to $\bar{g}_{a b}$ at this point. Furthermore, from (6.1) also it follows that (1.2) is satisfied on $\mathcal{U}_{S} \subset \bar{M} \times_{F} \widetilde{N}$, i.e. $\operatorname{rank}\left(S-\tau_{1} g\right) \leq 2$ on this set. In addition, if $\operatorname{rank}\left(S-\tau_{1} g\right)=2$ then $\bar{M} \times_{F} \widetilde{N}$ is a 2-quasi-Einstein manifold. Thus we have

Theorem 6.1. Let $\bar{M} \times{ }_{F} \widetilde{N}$ be the warped product manifold of a 2-dimensional semi-Riemannian manifold $(\bar{M}, \bar{g})$ and an $(n-2)$-dimensional semi-Riemannian manifold $(\widetilde{N}, \widetilde{g}), n \geq 4$, with a warping function $F$, and let $(\widetilde{N}, \widetilde{g})$ be an Einstein manifold, provided that $n \geq 5$. Then on $\mathcal{U}_{S} \subset \bar{M} \times_{F} \tilde{N}$ we have $\operatorname{rank}\left(S-\tau_{1} g\right) \leq 2$, where the function $\tau_{1}$ is defined by (6.2). Moreover, if $\operatorname{rank}\left(S-\tau_{1} g\right)=2$ on some open non-empty subset of $\mathcal{U}_{S}$ then $\bar{M} \times_{F} \widetilde{N}$ is a 2-quasi-Einstein manifold.

Let now $A$ be the $(0,2)$-tensor with the local components $A_{i j}$ defined by

$$
A_{i j}=S_{i j}-\tau_{1} g_{i j},
$$

where $\tau_{1}$ is the function defined by (6.2). Using (6.1) and (6.3) we get

$$
A_{a d}=S_{a d}-\tau_{1} g_{a d}, \quad A_{\alpha \beta}=S_{\alpha \beta}-\tau_{1} g_{\alpha \beta}=0, \quad A_{a \alpha}=0 .
$$

From (6.4) it follows immediately that $A_{a b}$ is proportional to $\bar{g}_{a b}$ if and only if $S_{a b}$ is proportional to $\bar{g}_{a b}$. Further, let $A^{2}$ be the $(0,2)$-tensor with the local components $A_{i j}^{2}=g^{r s} A_{i r} A_{j s}$. We have

$$
\begin{aligned}
A_{i j}^{2}= & S_{i j}^{2}-2 \tau_{1} S_{i j}+\tau_{1}^{2} g_{i j}, \quad A_{a d}^{2}=S_{a d}^{2}-2 \tau_{1} S_{a d}+\tau_{1}^{2} g_{a d}, \quad A_{\alpha \beta}^{2}=0, \quad A_{a \alpha}^{2}=0, \\
& \operatorname{tr}(A)=g^{r s} A_{r s}=\kappa-n \tau_{1}, \operatorname{tr}\left(A^{2}\right)=g^{r s} A_{r s}^{2}=\operatorname{tr}\left(S^{2}\right)-2 \kappa \tau_{1}+n \tau_{1}^{2}, \\
& \operatorname{tr}\left(A^{2}\right)-(\operatorname{tr}(A))^{2}=\operatorname{tr}\left(S^{2}\right)-\kappa^{2}+(n-1) \tau_{1}\left(2 \kappa-n \tau_{1}\right),
\end{aligned}
$$


and

$$
\begin{aligned}
& \operatorname{tr}\left(A^{2}\right)-(\operatorname{tr}(A))^{2}=g^{r s} A_{r s}^{2}-\left(g^{r s} A_{r s}\right)^{2} \\
= & \bar{g}^{11} A_{11}^{2}+2 \bar{g}^{12} A_{12}^{2}+\bar{g}^{22} A_{22}^{2}-\left(\bar{g}^{11} A_{11}+2 \bar{g}^{12} A_{12}+\bar{g}^{22} A_{22}\right)^{2} \\
= & \bar{g}^{11} g^{r s} A_{1 r} A_{1 s}+2 \bar{g}^{12} g^{r s} A_{1 r} A_{2 s}+\bar{g}^{22} g^{r s} A_{2 r} A_{2 s}-\left(\bar{g}^{11} A_{11}+2 \bar{g}^{12} A_{12}+\bar{g}^{22} A_{22}\right)^{2} \\
= & \bar{g}^{11}\left(\bar{g}^{11}\left(A_{11}\right)^{2}+2 \bar{g}^{12} A_{11} A_{12}+\bar{g}^{22}\left(A_{12}\right)^{2}\right)+\bar{g}^{22}\left(\bar{g}^{11}\left(A_{12}\right)^{2}+2 \bar{g}^{12} A_{12} A_{22}+\bar{g}^{22}\left(A_{22}\right)^{2}\right) \\
& +2 \bar{g}^{12}\left(\bar{g}^{11} A_{11} A_{12}+\bar{g}^{12} A_{11} A_{22}+\bar{g}^{12}\left(A_{12}\right)^{2}+\bar{g}^{22} A_{12} A_{22}\right)-\left(\bar{g}^{11} A_{11}+2 \bar{g}^{12} A_{12}+\bar{g}^{22} A_{22}\right)^{2} \\
= & -2\left(\bar{g}^{11} \bar{g}^{22}-\left(\bar{g}^{12}\right)^{2}\right)\left(A_{11} A_{22}-\left(A_{12}\right)^{2}\right),
\end{aligned}
$$

i.e.

$$
\operatorname{tr}\left(A^{2}\right)-(\operatorname{tr}(A))^{2}=-2(\operatorname{det}(\bar{g}))^{-1}\left(A_{11} A_{22}-\left(A_{12}\right)^{2}\right) .
$$

From (6.4) and (6.7) it follows that at every point of $x \in \mathcal{U}_{S} \subset \bar{M} \times_{F} \tilde{N}$ the conditions: $\operatorname{rank}(A)=2$ and $\operatorname{tr}\left(A^{2}\right)-(\operatorname{tr}(A))^{2} \neq 0$ are equivalent. Therefore on the set of at all points of $\mathcal{U}_{S}$ at which $\operatorname{rank}(A)=2$ we can define the function $\tau_{2}$ by

$$
\tau_{2}=\left(\operatorname{tr}\left(A^{2}\right)-(\operatorname{tr}(A))^{2}\right)^{-1} .
$$

We also note that in view of Lemma 2.2 we have

$$
\begin{aligned}
A_{a d}^{2} & =\operatorname{tr}(A) A_{a d}+\frac{1}{2}\left(\operatorname{tr}\left(A^{2}\right)-(\operatorname{tr}(A))^{2}\right) \bar{g}_{a d}, \\
Q\left(A, A^{2}\right)_{a b c d} & =-\frac{1}{2}\left(\operatorname{tr}\left(A^{2}\right)-(\operatorname{tr}(A))^{2}\right) Q(\bar{g}, A)_{a b c d} .
\end{aligned}
$$

We have

Theorem 6.2. Let $\bar{M} \times{ }_{F} \widetilde{N}$ be the warped product manifold of a 2-dimensional semi-Riemannian manifold $(\bar{M}, \bar{g})$ and an $(n-2)$-dimensional semi-Riemannian manifold $(\tilde{N}, \widetilde{g}), n \geq 4$, with a warping function $F$, and let $(\widetilde{N}, \widetilde{g})$ be an Einstein manifold, provided that $n \geq 5$. Moreover, let $V$ be the set of all points of $\mathcal{U}_{S} \cap \mathcal{U}_{C} \subset \bar{M} \times_{F} \widetilde{N}$ at which $S_{a d}$ is not proportional to $\bar{g}_{a d}$. Then on $V$ we have

$$
\begin{aligned}
R \cdot S & =\left(\phi_{1}-2 \tau_{1} \phi_{2}+\tau_{1}^{2} \phi_{3}\right) Q(g, S)+\left(\phi_{2}-\tau_{1} \phi_{3}\right) Q\left(g, S^{2}\right)+\phi_{3} Q\left(S, S^{2}\right), \\
\phi_{1} & =\frac{2 \tau_{1}-\bar{\kappa}}{2(n-2)}, \quad \phi_{2}=\frac{1}{n-2}, \quad \phi_{3}=\frac{\tau_{2}\left(2 \kappa-\bar{\kappa}-2(n-1) \tau_{1}\right)}{n-2} .
\end{aligned}
$$

The condition (3.3) holds on the set $\left(\mathcal{U}_{S} \cap \mathcal{U}_{C}\right) \backslash V$.

Proof. Let $A$ be the (0,2)-tensor defined by (6.4). Using now (2.4), (2.5), (4.1), (4.2), (6.1) and (6.4) we get

$$
\begin{aligned}
&-\frac{1}{2 F} T_{a d}=\frac{1}{n-2}\left(A_{a d}+\frac{2 \tau_{1}-\bar{\kappa}}{2} g_{a d}\right), \\
& R_{a b c d}=\frac{\bar{\kappa}}{2} G_{a b c d}, \quad R_{a \alpha \beta d}=\frac{1}{n-2}\left(A_{a d}+\frac{2 \tau_{1}-\bar{\kappa}}{2(n-2)} g_{a d}\right) g_{\alpha \beta}, \\
&(R \cdot A)_{a b c d}=\frac{\bar{\kappa}}{2} Q(g, A)_{a b c d}, \quad(R \cdot A)_{a \alpha \beta d}=\frac{1}{n-2}\left(A_{a d}^{2}+\frac{2 \tau_{1}-\bar{\kappa}}{2} A_{a d}\right) g_{\alpha \beta}, \\
&(R \cdot A)_{\alpha \beta \gamma \delta}=0, \quad Q(g, A)_{\alpha \beta \gamma \delta}=Q\left(g, A^{2}\right)_{\alpha \beta \gamma \delta}=Q\left(A, A^{2}\right)_{\alpha \beta \gamma \delta}=0 .
\end{aligned}
$$


Using the above presented relations and (6.9) we obtain on $V$ the following condition

$$
R \cdot A=\phi_{1} Q(g, A)+\phi_{2} Q\left(g, A^{2}\right)+\phi_{3} Q\left(A, A^{2}\right),
$$

where $\phi_{1}, \phi_{2}$ and $\phi_{3}$ are defined on $V$ by (6.11). Now (6.12), by (6.4) and (6.5), turns into (6.10). Finally, from (6.1) and the fact that $S_{a b}$ is proportional to $g_{a b}$ on $\left(\mathcal{U}_{S} \cap \mathcal{U}_{C}\right) \backslash V$ it follows that $T_{a b}$ also is proportional to $g_{a b}$ on this set. Therefore, in view of [20, Corollary 4.1], (3.3) holds on $\left(\mathcal{U}_{S} \cap \mathcal{U}_{C}\right) \backslash V$. The last remark completes proof.

\section{WARPED PRODUCT MANIFOLDS WITH 2-DIMENSIONAL BASE AND FIBRE OF CONSTANT CURVATURE}

We consider the warped product manifold $\bar{M} \times{ }_{F} \widetilde{N}$ of a 2-dimensional manifold $(\bar{M}, \bar{g})$ and an $(n-2)$-dimensional semi-Riemannian manifold $(\widetilde{N}, \widetilde{g}), n \geq 4$, with a warping function $F$, and let $(\widetilde{N}, \widetilde{g})$ be a space of constant curvature, provided that $n \geq 5$. Using Lemma 1.1, (4.1)-(4.4) and [22, eqs. (12)-(16)] we can check that the local components $C_{h i j k}$ of the tensor Ricci tensor $S$ and the Weyl conformal curvature tensor $C$ of $\bar{M} \times_{F} \widetilde{N}$ are expressed by

$$
\begin{aligned}
C_{a b c d} & =\frac{(n-3) \rho_{0}}{n-1} G_{a b c d}, \quad C_{\alpha b c \delta}=-\frac{(n-3) \rho_{0}}{(n-2)(n-1)} G_{\alpha b c \delta}, \\
C_{\alpha \beta \gamma \delta} & =\frac{2 \rho_{0}}{(n-2)(n-1)} G_{\alpha \beta \gamma \delta}, \quad C_{a b c \delta}=C_{a b \gamma \delta}=C_{a \beta \gamma \delta}=0,
\end{aligned}
$$

respectively, where

$$
\rho_{0}=\frac{\bar{\kappa}}{2}+\frac{\widetilde{\kappa}}{(n-3)(n-2) F}+\frac{\operatorname{tr}(T)}{2 F}-\frac{\Delta_{1} F}{4 F^{2}} .
$$

We also have

$$
\begin{aligned}
& F \tau_{1}+(n-3) \frac{\bar{\kappa}}{2} F+(n-2) \frac{\operatorname{tr}(T)}{2} \\
= & (n-3) \frac{\bar{\kappa}}{2} F+\frac{\widetilde{\kappa}}{n-2}+(n-3) \frac{\operatorname{tr}(T)}{2}-(n-3) \frac{\Delta_{1} F}{4 F} \\
= & (n-3) F\left(\frac{\bar{\kappa}}{2}+\frac{\widetilde{\kappa}}{(n-3)(n-2) F}+\frac{\operatorname{tr}(T)}{2 F}-\frac{\Delta_{1} F}{4 F^{2}}\right)=(n-3) F \rho_{0}=\frac{n-1}{2} F \rho,
\end{aligned}
$$

where

$$
\rho=\frac{2(n-3) \rho_{0}}{n-1}
$$

Now the condition (7.1), by (7.4), turns into

$$
\begin{aligned}
C_{a b c d} & =\frac{\rho}{2} G_{a b c d}, \quad C_{\alpha b c \delta}=-\frac{\rho}{2(n-2)} G_{\alpha b c \delta}, \\
C_{\alpha \beta \gamma \delta} & =\frac{\rho}{(n-3)(n-2)} G_{\alpha \beta \gamma \delta}, \quad C_{a b c \delta}=C_{a b \gamma \delta}=C_{a \beta \gamma \delta}=0 .
\end{aligned}
$$

Remark 7.1. Let $\bar{M} \times_{F} \widetilde{N}$ be the warped product manifold of a 2-dimensional manifold $(\bar{M}, \bar{g})$ and an $(n-2)$-dimensional semi-Riemannian manifold $(\widetilde{N}, \widetilde{g}), n \geq 4$, with a warping function $F$, and let $(\widetilde{N}, \widetilde{g})$ be a space of constant curvature, provided that $n \geq 5$. (i) From (17.1) it 
follows immediately that the manifold $\bar{M} \times_{F} \widetilde{N}$ is conformally flat if and only if the function $\rho_{0}$, defined by (7.2), vanishes on $\bar{M}$. (ii) We refer to [18, Lemma 3.3, Lemma 4.1, Lemma 4.3], [14, Example 5.4 (i), (ii)] and [73, Sections 4 and 5] for examples of conformally flat warped product manifolds $\bar{M} \times_{F} \widetilde{N}$, with $\operatorname{dim} \bar{M} \geq 2$. (iii) Recently warped product spacetimes $\bar{M} \times_{F} \widetilde{N}$, with $\operatorname{dim} \bar{M}=\widetilde{N}=2$, satisfying curvature conditions of pseudosymmetry type were studied in [40].

Theorem 7.1. Let $\bar{M} \times{ }_{F} \widetilde{N}$ be the warped product manifold of a 2-dimensional semi-Riemannian manifold $(\bar{M}, \bar{g})$ and an $(n-2)$-dimensional semi-Riemannian manifold $(\tilde{N}, \widetilde{g}), n \geq 4$, with a warping function $F$, and let $(\tilde{N}, \widetilde{g})$ be a space of constant curvature, provided that $n \geq 5$.

(i) The following three conditions are satisfied on the set $\mathcal{U}_{C} \subset \bar{M} \times_{F} \tilde{N}$ :

$$
C \cdot C=-\frac{\rho}{2(n-2)} Q(g, C),
$$

where $\rho$ is defined by (7.4), (1.8) with the function $L$ be defined by

$$
L=-\frac{n-2}{(n-1) \rho}\left(\bar{\kappa}\left(\tau_{1}+\frac{\operatorname{tr}(T)}{2 F}\right)+\frac{n-3}{4 F^{2}}\left(\operatorname{tr}\left(T^{2}\right)-(\operatorname{tr}(T))^{2}\right)\right),
$$

where $\tau_{1}$ is defined by (6.2), and (1.15) with $L_{C}=-\frac{\rho}{2(n-2)}$ and $L$ defined by (7.7).

(ii) Let $V$ be the set of all points of $\mathcal{U}_{S} \cap \mathcal{U}_{C} \subset \bar{M} \times_{F} \widetilde{N}$ at which $S_{a d}$ is not proportional to $\bar{g}_{a d}$. Then on $V$ we have:

$$
\begin{gathered}
C=-\frac{(n-1) \rho \tau_{2}}{(n-3)(n-2)}\left(\frac{n-2}{2} S \wedge S-\kappa g \wedge S+g \wedge S^{2}-\frac{\operatorname{tr}\left(S^{2}\right)-\kappa^{2}}{n-1} G\right), \\
R \cdot C+C \cdot R=Q(S, C)+\left(L-\frac{\rho}{2(n-2)}+\frac{n-3}{(n-2)(n-1) \rho \tau_{2}}\right) Q(g, C), \\
C \cdot R=-\frac{1}{(n-2)^{2}} Q\left(\left(\frac{\rho}{2}+(n-1) \rho \tau_{1}^{2} \tau_{2}\right) S-(n-1) \rho \tau_{1} \tau_{2} S^{2}, G\right) \\
\quad-\frac{(n-1) \rho \tau_{2}}{(n-2)^{2}} g \wedge Q\left(S, S^{2}\right)-\frac{\rho}{2(n-2)} Q(g, C), \\
R \cdot C=Q(S, C)+\left(L+\frac{n-3}{(n-2)(n-1) \rho \tau_{2}}\right) Q(g, C)+\frac{(n-1) \rho \tau_{2}}{(n-2)^{2}} g \wedge Q\left(S, S^{2}\right) \\
\quad+\frac{1}{(n-2)^{2}} Q\left(\left(\frac{\rho}{2}+(n-1) \rho \tau_{1}^{2} \tau_{2}\right) S-(n-1) \rho \tau_{1} \tau_{2} S^{2}, G\right) .
\end{gathered}
$$

On the set $\left(\mathcal{U}_{S} \cap \mathcal{U}_{C}\right) \backslash V$ the Weyl tensor $C$ is expressed by a linear combination of the KulkarniNomizu products $S \wedge S, g \wedge S$ and $g \wedge g$.

Proof. (i) Using (2.2) and (2.3) we can verify that the local components $(C \cdot C)_{h i j k l m}$ and $Q(g, C)_{h i j k l m}$ of the tensors $C \cdot C$ and $Q(g, C)$ which may not vanish are those related to

$$
\begin{aligned}
& (7.12)(C \cdot C)_{\alpha a b c d \beta}=-\frac{(n-1) \rho^{2}}{4(n-2)^{2}} g_{\alpha \beta} G_{d a b c}, \quad(C \cdot C)_{a \alpha \beta \gamma d \beta}=\frac{(n-1) \rho^{2}}{4(n-2)^{2}(n-3)} g_{a d} G_{\delta \alpha \beta \gamma}, \\
& (7.13) Q(g, C)_{\alpha a b c d \beta}=\frac{(n-1) \rho}{2(n-2)} g_{\alpha \beta} G_{d a b c}, \quad Q(g, C)_{a \alpha \beta \gamma d \delta}=-\frac{(n-1) \rho}{2(n-2)(n-3)} g_{a d} G_{\delta \alpha \beta \gamma}
\end{aligned}
$$


(cf. [15, eqs. (8)-(11)]). From (7.12) and (7.13) it follows that (1.9) holds on $\mathcal{U}_{C} \subset \bar{M} \times_{F} \tilde{N}$, where $L_{C}=-\frac{\rho}{2(n-2)}$ and $\rho$ is defined by (7.4).

We prove now that (1.8) is satisfied. First of all, we recall that necessary and sufficient conditions for warped products of two semi-Riemannian spaces of constant curvature satisfying that condition are given in [17]. In particular, when the base $(\bar{M}, \bar{g})$ is a 2-dimensional manifold, $(\tilde{N}, \widetilde{g})$ a space of constant curvature (when $n \geq 5$ ), then (1.8) holds on $\mathcal{U}_{C} \subset \bar{M} \times_{F} \tilde{N}$ if and only if

$$
\begin{aligned}
& \left(\left(\frac{\widetilde{\kappa}}{n-2}-\frac{1}{2}\left(\operatorname{tr}(T)+\frac{n-3}{2 F} \Delta_{1} F\right)\right)\left(\frac{\bar{\kappa}}{2}+\frac{L}{n-2}\right)+\frac{n-3}{n-2} \frac{F L \bar{\kappa}}{2}\right) \bar{G}_{d a b c} \\
= & \frac{n-3}{4 F}\left(T_{a b} T_{c d}-T_{a c} T_{b d}\right)-\left(\frac{\bar{\kappa}}{4}+\frac{L}{2}\right)\left(\bar{g}_{a b} T_{c d}+\bar{g}_{c d} T_{a b}-\bar{g}_{a c} T_{b d}-\bar{g}_{b d} T_{a c}\right)
\end{aligned}
$$

on $\mathcal{U}_{C}$ (cf., [17, Section 7, eq. (40)]). Applying in (7.14) the relation (6.2) and the definitions of the tensors $g \wedge T$ and $T \wedge T$ we obtain

$$
\left(\left(\bar{\kappa}+\frac{2 L}{n-2}\right) F \tau_{1}+\frac{n-3}{n-2} F L \bar{\kappa}\right) \bar{G}_{d a b c}=\frac{n-3}{2 F} \frac{1}{2}(T \wedge T)_{d a b c}-\left(\frac{\bar{\kappa}}{2}+L\right)(g \wedge T)_{d a b c} .
$$

Thie last equation, together with

$$
\begin{aligned}
(g \wedge T)_{1221} & =\operatorname{tr}(T) G_{1221}=\operatorname{tr}(T) \operatorname{det}(\bar{g}) \\
\frac{1}{2}(T \wedge T)_{1221} & =T_{11} T_{22}-\left(T_{12}\right)^{2}=-\frac{1}{2} \operatorname{det}(\bar{g})\left(\operatorname{tr}\left(T^{2}\right)-(\operatorname{tr}(T))^{2}\right),
\end{aligned}
$$

leads to

$$
\begin{gathered}
\left(\left(\bar{\kappa}+\frac{2 L}{n-2}\right) F \tau_{1}+\frac{n-3}{n-2} F L \bar{\kappa}\right) \operatorname{det}(\bar{g}) \\
=-\frac{n-3}{2 F} \frac{1}{2}\left(\operatorname{tr}\left(T^{2}\right)-(\operatorname{tr}(T))^{2}\right) \operatorname{det}(\bar{g})-\left(\frac{\bar{\kappa}}{2}+L\right) \operatorname{tr}(T) \operatorname{det}(\bar{g}) \\
\left(\left(\bar{\kappa}+\frac{2 L}{n-2}\right) F \tau_{1}+\frac{n-3}{n-2} F L \bar{\kappa}\right)=-\frac{n-3}{2 F} \frac{1}{2}\left(\operatorname{tr}\left(T^{2}\right)-(\operatorname{tr}(T))^{2}\right)-\left(\frac{\bar{\kappa}}{2}+L\right) \operatorname{tr}(T), \\
\left(\frac{2}{n-2} F \tau_{1}+\frac{n-3}{n-2} F \bar{\kappa}+\operatorname{tr}(T)\right) L=-F \bar{\kappa} \tau_{1}-\frac{n-3}{4 F}\left(\operatorname{tr}\left(T^{2}\right)-(\operatorname{tr}(T))^{2}\right)-\frac{\bar{\kappa}}{2} \operatorname{tr}(T), \\
=-\frac{n-2}{2}\left(F \bar{\kappa}\left(\tau_{1}+\frac{\operatorname{tr}(T)}{2 F}\right)+\frac{n-3}{4 F}\left(\operatorname{tr}\left(T^{2}\right)-(\operatorname{tr}(T))^{2}\right)\right) .
\end{gathered}
$$

This, by making use of (6.2), (7.2) and (7.4), turns into

$$
(n-1) \rho L=-(n-2)\left(\bar{\kappa}\left(\tau_{1}+\frac{\operatorname{tr}(T)}{2 F}\right)+\frac{n-3}{4 F^{2}}\left(\operatorname{tr}\left(T^{2}\right)-(\operatorname{tr}(T))^{2}\right)\right),
$$


which, together with (7.3) and (7.4), yields (7.7). Now Theorem 3.3(ii) completes the proof of (i). (ii) First we prove that the following relation is satisfied on $V$ :

$$
\begin{gathered}
C=\frac{\phi_{1}}{2} A \wedge A+\phi_{2} g \wedge A+\phi_{3} G+\phi_{4} g \wedge A^{2} \\
=-\frac{(n-1) \rho \tau_{2}}{(n-3)(n-2)}\left(\frac{n-2}{2} A \wedge A-\operatorname{tr}(A) g \wedge A+g \wedge A^{2}-\frac{1}{(n-1) \tau_{2}} G\right), \\
\phi_{1}=-\frac{(n-1) \rho \tau_{2}}{n-3}, \quad \phi_{2}=\frac{(n-1) \rho \tau_{2} \operatorname{tr}(A)}{(n-3)(n-2)} \\
\phi_{3}=\frac{\rho}{(n-3)(n-2)}, \quad \phi_{4}=-\frac{(n-1) \rho \tau_{2}}{(n-3)(n-2)},
\end{gathered}
$$

where the $(0,2)$-tensor $A$ is defined on $V$ by (6.4) . Let $B$ be the $(0,4)$-tensor defined on $V$ by

$$
B=C-\frac{\phi_{1}}{2} A \wedge A-\phi_{2} g \wedge A-\phi_{3} G-\phi_{4} g \wedge A^{2},
$$

where $\phi_{1}, \ldots, \phi_{4}$ are some functions on $V$. Evidently, $B$ is generalized curvature tensor. Let $B_{\text {hijk }}$ be the local components of $B$. We have

$$
\begin{aligned}
B_{h i j k}= & C_{h i j k}-\phi_{1}\left(A_{h k} A_{i j}-A_{h j} A_{i k}\right)-\phi_{2}\left(g_{h k} A_{i j}+g_{i j} A_{h k}-g_{h j} A_{i k}-g_{i k} A_{h j}\right) \\
& -\phi_{3}\left(g_{h k} g_{i j}-g_{h j} g_{i k}\right)-\phi_{4}\left(g_{h k} A_{i j}^{2}+g_{i j} A_{h k}^{2}-g_{h j} A_{i k}^{2}-g_{i k} A_{h j}^{2}\right) .
\end{aligned}
$$

It is clear that $B$ vanish at a point $x \in V$ if and only if

$$
\begin{aligned}
C_{h i j k}= & \phi_{1}\left(A_{h k} A_{i j}-A_{h j} A_{i k}\right)+\phi_{2}\left(g_{h k} A_{i j}+g_{i j} A_{h k}-g_{h j} A_{i k}-g_{i k} A_{h j}\right) \\
& +\phi_{3}\left(g_{h k} g_{i j}-g_{h j} g_{i k}\right)+\phi_{4}\left(g_{h k} A_{i j}^{2}+g_{i j} A_{h k}^{2}-g_{h j} A_{i k}^{2}-g_{i k} A_{h j}^{2}\right)
\end{aligned}
$$

at $x$. We note that from (6.4) and (7.1) it follows immediately that the local components $B_{h i j k}$ of the tensor $B$ which may not vanish identically are the following: $B_{a b c d}, B_{\alpha b c \delta}$ and $B_{\alpha \beta \gamma \delta}$. Thus we see that $B=0$ at $x$ if and only if

$$
\begin{aligned}
& \left(\frac{\rho}{2}-\phi_{3}\right) G_{a b c d}=\phi_{1}\left(A_{a d} A_{b c}-A_{a c} A_{b d}\right)+\phi_{2}\left(g_{a d} A_{b c}+b_{b c} A_{a d}-g_{a c} A_{b d}-g_{b d} A_{a c}\right) \\
& +\phi_{4}\left(g_{a d} A_{b c}^{2}+b_{b c} A_{a d}^{2}-g_{a c} A_{b d}^{2}-g_{b d} A_{a c}^{2}\right) \\
& -\left(\frac{\rho}{2(n-2)}+\phi_{3}\right) g_{b c} g_{\alpha \delta}=\phi_{2} A_{b c} g_{\alpha \delta}+\phi_{4} A_{b c}^{2} g_{\alpha \delta} \\
& \left(\frac{\rho}{(n-3)(n-2)}-\phi_{3}\right) G_{\alpha \beta \gamma \delta}=0
\end{aligned}
$$

at $x$. Further, (7.17), by (6.9), is equivalent to

$$
\begin{aligned}
& \phi_{1}\left(A_{11} A_{22}-A_{12} A_{12}\right)=\frac{(n-1) \rho}{2(n-3)} G_{1221}, \\
& -\left(\phi_{2}+\operatorname{tr}(A) \phi_{4}\right) A_{b c}=\left(\frac{(n-1) \rho}{2(n-3)(n-2)}+\frac{1}{2}\left(\operatorname{tr}\left(A^{2}\right)-(\operatorname{tr}(A))^{2}\right) \phi_{4}\right) g_{b c} \\
& \phi_{3}=\frac{\rho}{(n-3)(n-2)} .
\end{aligned}
$$


But this, together with (6.7), (6.8) and the fact that $A_{a b}$ is not proportional to $g_{a b}$, leads immediately to (7.16).

From (7.15), by (6.3), (6.5), (6.6) and (7.16), we get (7.8). Now (1.15), together with (7.7) and (7.8), yields (7.9) . Using (1.5), (2.14) and (7.6) we obtain

$$
\begin{aligned}
C \cdot R= & C \cdot\left(C+\frac{1}{n-2} g \wedge S-\frac{\kappa}{(n-2)(n-1)} G\right)=C \cdot C+\frac{1}{n-2} g \wedge(C \cdot S) \\
= & -\frac{1}{(n-2)^{2}} g \wedge Q\left(g,\left(\frac{\rho}{2}+(n-1) \rho \tau_{1}^{2} \tau_{2}\right) S-(n-1) \rho \tau_{1} \tau_{2} S^{2}\right) \\
& -\frac{(n-1) \rho \tau_{2}}{(n-2)^{2}} g \wedge Q\left(S, S^{2}\right)-\frac{\rho}{2(n-2)} Q(g, C)
\end{aligned}
$$

and in a consequence (7.10). From (17.9) and (17.10) it follows immediately (7.11).

From (6.1) and the fact that $S_{a b}$ is proportional to $g_{a b}$ on $\left(\mathcal{U}_{S} \cap \mathcal{U}_{C}\right) \backslash V$ it follows that $T_{a b}$ also is proportional to $g_{a b}$ on this set. Therefore, in view of Theorem 5.4, (1.7) holds on $\left(\mathcal{U}_{S} \cap \mathcal{U}_{C}\right) \backslash V$. Now using (1.5) and (1.7) we can express the tensor $C$ by a linear combination of the Kulkarni-Nomizu products $S \wedge S, g \wedge S$ and $g \wedge g$. The last remark completes proof of (ii).

Remark 7.1. (i) Let the curvature tensor $R$ of a semi-Riemannian manifold $(M, g), n \geq 4$, satisfies

$$
R=\frac{\phi_{1}}{2} S \wedge S+\phi_{2} g \wedge S+\phi_{3} G+\phi_{4} g \wedge S^{2}
$$

on $\mathcal{U}_{S} \cap \mathcal{U}_{C} \subset M$, where $\phi_{1}, \phi_{2}, \ldots, \phi_{4}$ are some functions on this set. Evidently, if (1.4) holds at a point of $\mathcal{U}_{S} \cap \mathcal{U}_{C}$ then (17.18) reduces to (1.7) at this point. We can prove that if the tensor $S^{3}$ is not a linear combination of $g, S$ and $S^{2}$ at a point $\mathcal{U}_{S} \cap \mathcal{U}_{C}$ then the decomposition (7.18) is unique at this point. We also note that (7.18), by (1.5), yields

$$
C=\frac{\phi_{1}}{2} S \wedge S+\left(\phi_{2}-\frac{1}{n-2}\right) g \wedge S+\left(\phi_{3}+\frac{\kappa}{(n-2)(n-1)}\right) G+\phi_{4} g \wedge S^{2} .
$$

(ii) Warped product manifolds $\bar{M} \times_{F} \tilde{N}, \operatorname{dim} \bar{M}=1$, satisfying (17.18) are investigated in [34].

Example 7.1. (i) Let $\bar{M}_{1}=\left\{(v, r) \in \mathbb{R}^{2}: r>0\right\}$, resp., $\bar{M}_{2}=\left\{(u, r) \in \mathbb{R}^{2}: r>0\right\}$, be an open connected non-empty subset of $\mathbb{R}^{2}$ and let on $\bar{M}_{1}$, resp., $\bar{M}_{2}$, the metric tensor $\bar{g}_{1}$, resp., $\bar{g}_{2}$, be defined by

$$
\bar{g}_{1 a b} d x^{a} d x^{b}=-f_{1} d v^{2}+2 d v d r, \quad \bar{g}_{2 a b} d x^{a} d x^{b}=-f_{2} d u^{2}-2 d u d r,
$$

where $x^{1}=v, x^{2}=r$ and $f_{1}=f_{1}(v, r)$, resp., $x^{1}=u, x^{2}=r$ and $f_{2}=f_{2}(u, r)$, is a smooth function on $\bar{M}_{1}$, resp., $\bar{M}_{2}$, and $a, b=1,2$. We consider the warped product manifold $\bar{M}_{i} \times_{F} \tilde{N}$, $i=1,2$, of $\left(\bar{M}_{i}, \bar{g}_{i}\right), i=1,2$, and the 2-dimensional standard unit sphere $(\widetilde{N}, \widetilde{g})$ with the warping function $F=F(r)=r^{2}$. (ii) (a) According to [7, Section 29.5.2] (see also [63, Section 9.5]) the Vaidya metrics form a simple class of timedependent generalizations of the Schwarzschild metric [87]. They can be obtained from the Schwarzschild metric written in ingoing or outgoing Eddington-Finkelstein coordinates by replacing the constant mass $m$ by a mass function $m(v)$ or $m(u)$ depending on an advanced or retarded time coordinate. The metrics of the warped products manifolds $\bar{M}_{i} \times_{F} \tilde{N}, i=1,2$, defined in (i), provided that $f_{1}(v, r)=1-\frac{2 m(v)}{r}$, 
resp., $f_{2}(u, r)=1-\frac{2 m(u)}{r}$, are the Vaidya metrics (see, e.g., [7, eq. (29.15)] and [63, eq. (9.32)]). (b) The metric of $\bar{M}_{1} \times_{F} \widetilde{N}$, resp., $\bar{M}_{2} \times_{F} \widetilde{N}$, is called the generalized Vaidya ingoing metric, resp., outgoing metric (see, e.g. [7, eq. (39.16)]. In particular, the metric of $\bar{M}_{1} \times_{F} \widetilde{N}$ with the function $f=f(v, r)=f_{1}(v, r)$ defined by $f(v, r)=1-\frac{2 m(v)}{r}-\frac{\Lambda r^{2}}{3}, \Lambda=$ const., $f(v, r)=1-\frac{2 m(v)}{r}-\frac{q^{2}}{r^{2}}, q=$ const., $f(v, r)=1-\frac{2 m(v)}{r}-\frac{q^{2}(v)}{r^{2}}$, respectively, is named the Vaidya-Kottler, the Vaidya-Reissner-Nordstrøm and the Vaidya-Bonnor ingoing metric, respectively, (see, e.g. [7, eqs. (39.18), (39.19), (39.20)]). (iii) (a) For the manifold $\bar{M}_{1} \times_{F} \widetilde{N}$, with $f_{1}(v, r)=1-\frac{2 m(v)}{r}$, we have: $S_{v v}=\frac{2 m^{\prime}}{r^{2}}, m^{\prime}=\frac{d m}{d v}, m=m(v)$, and $S_{h k}=0$, if $h \neq v$ or $k \neq v, S^{2}=0, \kappa=0, S \cdot R=\frac{2 m}{r^{3}} g \wedge S, C \neq 0$, in particular $C_{v r r v}=-\frac{2 m}{r^{3}}$. Moreover,

$$
C \cdot C=R \cdot R-Q(S, R)=\frac{1}{2}(R \cdot C+C \cdot R-Q(S, C))=-\frac{m}{r^{3}} Q(g, C) .
$$

(b) For the manifold $\bar{M}_{2} \times_{F} \tilde{N}$, with $f_{2}(u, r)=1-\frac{2 m(u)}{r}$, we have: $S_{u u}=-\frac{2 m^{\prime}}{r^{2}}, m^{\prime}=\frac{d m}{d u}$, $m=m(u)$, and $S_{h k}=0$, if $h \neq u$ or $k \neq u, S^{2}=0, \kappa=0, S \cdot R=\frac{2 m}{r^{3}} g \wedge S, C \neq 0$, in particular $C_{\text {urru }}=-\frac{2 m}{r^{3}}$. Moreover, we also have (7.19) (with $m=m(u)$ ). (iv) For the metric of the manifold $\bar{M}_{1} \times_{F} \tilde{N}$, with the function $f=f(v, r)=f_{1}(v, r)$, we have

$$
\begin{aligned}
& S_{v v}= \frac{1}{r^{2}}\left(\frac{r^{2}}{2} f_{r r}^{\prime \prime}+r f_{r}^{\prime}-\frac{r}{f} f_{v}^{\prime}\right) g_{v v}, \quad S_{v r}=-\frac{1}{r^{2}}\left(\frac{r^{2}}{2} f_{r r}^{\prime \prime}+r f_{r}^{\prime}\right) g_{v r}, \\
& S_{\alpha \beta}= \tau_{1} g_{\alpha \beta}, \quad \tau_{1}=\frac{1}{r^{2}}\left(-r f_{r}^{\prime}-f+1\right), \quad \kappa=-\frac{2}{r^{2}}\left(\frac{r^{2}}{2} f_{r r}^{\prime \prime}+2 r f_{r}^{\prime}+f-1\right), \\
& A_{v v}=S_{v v}-\tau_{1} g_{v v}=\frac{1}{r^{2}}\left(\frac{r^{2}}{2} f_{r r}^{\prime \prime}+2 r f_{r}^{\prime}+f-1-\frac{r}{f} f_{v}^{\prime}\right) g_{v v}, \\
& A_{v r}=S_{v r}-\tau_{1} g_{v r}=\frac{1}{r^{2}}\left(-\frac{r^{2}}{2} f_{r r}^{\prime \prime}+f-1\right) g_{v r}, \\
& A_{r r}=S_{r r}-\tau_{1} g_{r r}=0, \quad A_{\alpha \beta}=S_{\alpha \beta}-\tau_{1} g_{\alpha \beta}=0,
\end{aligned}
$$

where $f_{r r}^{\prime \prime}=\frac{\partial^{2} f}{\partial r^{2}}, f_{r}^{\prime}=\frac{\partial f}{\partial r}$ and $f_{v}^{\prime}=\frac{\partial f}{\partial v}$. We set $\tau_{3}=\frac{r^{2}}{2} f_{r r}^{\prime \prime}-r f_{r}^{\prime}+f-1$. Now we can state that $\bar{M}_{1} \times_{F} \widetilde{N}$ is a conformally flat manifold if and only if the function $\tau_{3}$ is a zero function. Furthermore, on $U_{C} \subset \bar{M}_{1} \times_{F} \widetilde{N}$ we have (7.5) and (17.6), with $n=4$ and $\rho=-\frac{2}{3} \tau_{3} r^{-2}$, as well as (1.8) with $L=\left((f-1) f_{r r}^{\prime \prime}-\frac{1}{2}\left(f_{r}^{\prime}\right)^{2}\right) \tau_{3}^{-1}$. We also note that $\bar{M}_{1} \times_{F} \tilde{N}$ is an Einstein manifold if and only if the function $f$ satisfies on $\bar{M}_{1}$ the following system of differential equations

$$
\frac{r^{2}}{2} f_{r r}^{\prime \prime}-f+1=0, \quad \frac{r^{2}}{2} f_{r r}^{\prime \prime}+2 r f_{r}^{\prime}-\frac{r}{f} f_{v}^{\prime}+f-1=0 .
$$

It is easy to see that at every point of $\bar{M}_{1} \times_{F} \widetilde{N}$ we have rank $A=2$ if and only if at every point of $\bar{M}_{1}$ we have $\frac{r^{2}}{2} f_{r r}^{\prime \prime}+f-1 \neq 0$. Finally, $A_{a b}$ is proportional to $g_{a b}$ at a point of $\bar{M}_{1} \times_{F} \tilde{N}$ if and only if at this point we have $r f_{r r}^{\prime \prime}+2 f_{r}^{\prime}-\frac{1}{f} f_{v}^{\prime}=0$.

Example 7.2. (i) Let $\bar{M}_{1}=\left\{(u, r) \in \mathbb{R}^{2}: r-2 m>0\right.$ (or $\left.\left.r-2 m<0\right)\right\}$ be an open connected non-empty subset of $\mathbb{R}^{2}$ and let on $\bar{M}_{1}$ the metric tensor $\bar{g}$ be defined by

$$
\bar{g}_{u u} d u^{2}+2 \bar{g}_{u r} d u d r+\bar{g}_{r r} d r^{2}=-\exp (2 \beta) f d u^{2}+2 \exp (\beta) d u d r
$$


where $x^{1}=u, x^{2}=r, f=1-\frac{2 m}{r}$, and $m=m(u, r)$ and $\beta=\beta(u, r)$ are some smooth functions on $\bar{M}$. Further, let $\widetilde{g}$ be the standard metric on the 2-dimensional unit sphere $\tilde{N}=S^{2}(1)$. We denote by $g=\bar{g} \times_{F} \widetilde{g}$, where $F=F(r)=r^{2}$, the warped product metric of $\bar{M} \times_{F} \widetilde{N}$. The metric $g$ is said to be the general spherically symmetric metric in advanced EddingtonFilkenstein coordinates, see, e.g., [80, Section 4.1]. (ii) The local components of the Ricci tensor $S$ of $\bar{M} \times_{F} \tilde{N}$ which may not vanish identically are the following

$$
\begin{aligned}
S_{u u}= & \frac{1}{r^{2}(r-2 m)}\left(-2 r \exp (-\beta) m_{u}^{\prime}-\left(-3 r^{2}+6 r m\right) \beta_{r}^{\prime} m_{r}^{\prime}-\left(-r^{2}+2 r m\right) m_{r r}^{\prime \prime}\right. \\
& -\left(r^{3}-2 r^{2} m\right) \exp (-\beta) \beta_{u r}^{\prime \prime}-\left(2 r^{2}-5 r m+2 m^{2}\right) \beta_{r}^{\prime} \\
& \left.-\left(r^{3}-4 r^{2} m+4 r m^{2}\right)\left(\left(\beta_{r}^{\prime}\right)^{2}+\beta_{r r}^{\prime \prime}\right)\right) g_{u u}, \\
S_{u r}= & \frac{1}{r^{2}}\left(-r^{2} \exp (-\beta) \beta_{u r}^{\prime \prime}+3 r m_{r}^{\prime} \beta_{r}^{\prime}+r m_{r r}^{\prime \prime}+(-2 r+m) \beta_{r}^{\prime}+\left(-r^{2}+2 r m\right)\left(\left(\beta_{r}^{\prime}\right)^{2}+\beta_{r r}^{\prime \prime}\right)\right) g_{u r}, \\
S_{r r}= & \frac{2}{r} \beta_{r}^{\prime}, \quad S_{\phi \phi}=\tau_{1} g_{\phi \phi}, \quad S_{\theta \theta}=\tau_{1} g_{\theta \theta}, \quad \tau_{1}=\frac{1}{r^{2}}\left(2 m_{r}^{\prime}-(r-2 m) \beta_{r}^{\prime}\right),
\end{aligned}
$$

where $g_{\phi \phi}=r^{2} \widetilde{g}_{\phi \phi}, \widetilde{g}_{\phi \phi}=1, g_{\theta \theta}=r^{2} \widetilde{g}_{\theta \theta}, \widetilde{g}_{\theta \theta}=\sin ^{2} \phi$ and $m_{r}^{\prime}=\frac{\partial m}{\partial r}, m_{r r}^{\prime \prime}=\frac{\partial^{2} m}{\partial r^{2}}, m_{u}^{\prime}=\frac{\partial m}{\partial u}, \beta_{r}^{\prime}=$ $\frac{\partial \beta}{\partial r}, \beta_{r r}^{\prime \prime}=\frac{\partial^{2} \beta}{\partial r^{2}}, \beta_{u r}^{\prime \prime}=\frac{\partial^{2} \beta}{\partial u \partial r}$. (iii) In the class of the general spherically symmetric metrics $g$ we also have non-Einstein metrics. For instance, from the above formulas it follows immediately that the metrics $g$ with $S_{r r} \neq 0$, i.e. with $\beta_{r}^{\prime} \neq 0$, are non-Einstein metrics. Moreover, for such metrics $S_{a b}$ are non-proportional to $g_{a b}, a, b=1,2$. Some general spherically symmetric $g$ also are non-conformally flat metrics. Namely, the metrics $g$ satisfying

$$
\begin{aligned}
& r^{3}\left(\exp (-\beta) \beta_{r u}^{\prime \prime}+\left(\beta_{r}^{\prime}\right)^{2}+\beta_{r r}^{\prime \prime}\right)-r^{2}\left(m_{r r}^{\prime \prime}+\beta_{r}^{\prime}+2 m\left(\beta_{r}^{\prime}\right)^{2}+2 m \beta_{r r}^{\prime \prime}+3 \beta_{r}^{\prime} m_{r}^{\prime}\right) \\
& -r\left(5 m \beta_{r}^{\prime}+4 m_{r}^{\prime}\right)-6 m=0
\end{aligned}
$$

are non-conformally flat. This means that for some general spherically symmetric metrics $g$ the set $V$, defined in Theorem 7.1, is a non-empty subset of $\mathcal{U}_{S} \cap \mathcal{U}_{C} \subset \bar{M} \times{ }_{F} \widetilde{N}$.

Example 7.3. (i) Let $\bar{M}=\left\{(t, r) \in \mathbb{R}^{2}: t>0\right.$ and $\left.r>0\right\}$ be an open connected non-empty subset of $\mathbb{R}^{2}$ and let on $\bar{M}$ the metric tensor $\bar{g}$ be defined by $\bar{g}_{a b} d x^{a} d x^{b}=d t^{2}+R^{2}(t) d r^{2}$, $a, b=1,2$, where $x^{1}=t, x^{2}=r$, and $R=R(t)$ is a smooth positive (or negative) function on $\bar{M}$. Let $\bar{M} \times_{F} \widetilde{N}$ be the warped product manifold of the manifold $(\bar{M}, \bar{g})$ and the 2-dimensional standard unit sphere $(\widetilde{N}, \widetilde{g})$ with the warping function $F=F(t, r)=(f(r) R(t))^{2}$, where $f=f(r)$ is a smooth positive (or negative) function on $\bar{M}$. We denote by $g=\bar{g} \times_{F} \widetilde{g}$ the metric of $\bar{M} \times{ }_{F} \tilde{N}$. We mention that the metric $g$ was considerd in [65, Section 4] (see also [66, Section 6]). (ii) We set $\rho_{0}=\left(f f_{r r}^{\prime \prime}-\left(f_{r}^{\prime}\right)^{2}+1\right)(f R)^{-2}$, where $f_{r}^{\prime}=\frac{d f}{d r}$ and $f_{r r}^{\prime \prime}=\frac{d f_{r}^{\prime}}{d r}$. We can check that the Weyl conformal curvature tensor $C$ of $g$ is a zero tensor if and only if $\rho_{0}=0$ on $\bar{M}$. Further, we have $S_{12}=S_{21}=0, S_{11}=\lambda_{1} g_{11}, S_{22}=\lambda_{2} g_{22}, S_{\alpha \beta}=\tau_{1} g_{\alpha \beta}=(f R)^{2} \tau_{1} \widetilde{g}_{\alpha \beta}$, $\alpha, \beta=3,4$, where

$$
\begin{aligned}
& \lambda_{1}=-3 R_{t t}^{\prime \prime} R^{-1}, \quad R_{t}^{\prime}=\frac{d R}{d t}, \quad R_{t t}^{\prime \prime}=\frac{d R_{t}^{\prime}}{d t}, \quad \lambda_{2}=-\left(f R R_{t t}^{\prime \prime}+2 f\left(R_{t}^{\prime}\right)^{2}+2 f_{r r}^{\prime \prime}\right) f^{-1} R^{-2}, \\
& \tau_{1}=-\left(f^{2} R R_{t t}^{\prime \prime}+2 f^{2}\left(R_{t}^{\prime}\right)^{2}+f f_{r r}^{\prime \prime}+\left(f_{r}^{\prime}\right)^{2}-1\right)(f R)^{-2}=\lambda_{2}+\rho_{0}
\end{aligned}
$$


and $\widetilde{g}_{\alpha \beta}$ are the local components of the metric $\widetilde{g}$. (iii) From (ii) it follows that $\lambda_{1}=\lambda_{2}$ if and only if $R R_{r r}^{\prime \prime}-\left(R_{r}^{\prime}\right)^{2}=c_{1}$ and $f_{r r}^{\prime \prime}=c_{1} f$ and $c_{1}=$ const. on $\bar{M}$. (iv) If $\rho_{0}$ is non-zero at a point of $\mathcal{U}_{C} \subset \bar{M} \times_{F} \widetilde{N}$ then in view of (ii) $S-\frac{\kappa}{4} g \neq 0$ at this point. Thus we have $\mathcal{U}_{C} \subset \mathcal{U}_{S} \subset \bar{M} \times_{F} \tilde{N}$. Moreover, the following relations are satisfied on $\mathcal{U}_{C}$

$$
\begin{aligned}
& R \cdot R-Q(S, R)=-\frac{2}{3} \rho_{0} Q(g, C), C \cdot C=-\frac{1}{6} \rho_{0} Q(g, C), \\
& R \cdot C+C \cdot R=Q(S, C)-\frac{1}{6}\left(\kappa+2 \rho_{0}\right) Q(g, C) .
\end{aligned}
$$

(v) If $\lambda=\lambda_{1}=\lambda_{2}$ at a point of $\mathcal{U}_{C}$ then $S_{a b}=\lambda g_{a b}$, and by (4.2), $T_{a b}=\frac{\operatorname{tr} T}{2} g_{a b}$ at this point. Let $V$ be the set of all points of $\mathcal{U}_{C}$ having this property. From (iii) it follows that for some functions $f$ and $R$ the set $V$ is non-empty and in view of [47, Theorem 4.1] we can state that (1.7) holds on this set. (vi) If $\lambda_{1} \neq \lambda_{2}$ at a point of $\mathcal{U}_{C}$ then $S_{a b}$ is not proportional to $g_{a b}$ at this point. Let $V$ be the set of all points of $\mathcal{U}_{C}$ having this property. From (iii) it follows that for some functions $f$ and $R$ the set $V$ is non-empty and in view of Theorem 7.1(ii) we can state that (7.8)-(7.11) hold on this set.

\section{REFERENCES}

[1] L. Alías, A. Romero and M. Sánchez, Compact spacelike hypersurfaces of constant mean curvature in generalized Robertson-Walker spacetimes, in: Geometry and Topology of Submanifolds, VII, World Sci., River Edge, NJ, 1991, 67-70.

[2] L. Alías, A. Romero and M. Sánchez, Spacelike hypersurfaces of constant mean curvature and CalabiEinstein type problems, Tôhoku Math. J. 49 (1997), 337-345.

[3] K. Arslan, R. Deszcz, R. Ezentaș, M. Hotloś and C. Murathan, On certain pseudosymmetry type warped products, Turkish J. Math. 38 (2014), 353-373.

[4] M. Belkhelfa, R. Deszcz and L. Verstraelen, Pseudosymmetry of 3-dimensional D'Atri space, Kyungpook Math. J. 46 (2006), 367-376.

[5] A.L. Besse, Einstein Manifolds, Ergeb. Math. Grenzgeb., 3. Folge, Bd. 10, Springer-Verlag, Berlin, Heidelberg, New York, 1987.

[6] R.L. Bishop and B. O'Neill, Manifolds of negative curvature, Trans. Amer. Math. Soc. 145 (1969), 1-49.

[7] M. Blau, Lecture Notes on General Relativity, 2014. http:// www.blau.itp.unibe.ch/Lecturenotes.html

[8] G. Calvaruso and D. Perrone, Geometry of Kaluza-Klein metrics on the sphere $S^{3}$, Annali di Mat. 192 (2013), 879-900.

[9] B.-Y. Chen, Some pinching and classification theorems for minimal submanifolds, Arch. Math. (Basel) 60 (1993), 568-578.

[10] B.-Y. Chen, $\delta$-invariants, inequalities of submanifolds and their applications, Chap. 2, Topics in Diff. Geom., Rom. Acad. Sci., Bucarest (2008).

[11] B.-Y. Chen, Geometric and topological obstructions to various immersions in submanifold theory and some related open problems, Kragujevac J. Math. 39 (2015), in print.

[12] J. Chojnacka-Dulas, R. Deszcz, M. Głogowska and M. Prvanović, On warped product manifolds satisfying some curvature conditions, J. Geom. Phys. 74 (2013), 328-341.

[13] F. Defever and R. Deszcz, On semi-Riemannian manifolds satisfying the condition $R \cdot R=Q(S, R)$, in: Geometry and Topology of Submanifolds, III, World Sci., River Edge, NJ, 1991, 108-130.

[14] F. Defever and R. Deszcz, On warped product manifolds satisfying a certain curvature condition, Atti Accad. Peloritana Pericolanti, Cl. Sci. Fis. Mat. Nat. 69 (1991), 213-236.

[15] F. Defever and R. Deszcz, On Riemannian manifolds satisfying a certain curvature condition imposed on the Weyl curvature tensor, Acta Univ. Palack. Olomuc. Fac. Rerum Natur. Math. 110 (32) (1993), 27-34.

[16] F. Defever, R. Deszcz, M. Hotloś, M. Kucharski and Z. Șentürk, Generalisations of Robertson-Walker spaces, Annales Univ. Sci. Budapest. Eötvös Sect. Math. 43 (2000), 13-24. 
[17] F. Defever, R. Deszcz and M. Prvanović, On warped product manifolds satisfying some curvature condition of pseudosymmetry type, Bull. Greek Math. Soc. 36 (1994), 43-67.

[18] J. Deprez, R. Deszcz and L. Verstraelen, Examples of pseudosymmetric conformally flat warped products, Chinese J. Math. 17 (1989), 51-65.

[19] J. Deprez, W. Roter and L. Verstraelen, Conditions on the projective curvature tensor of conformally flat Riemannian manifolds, Kyungpook Math. J. 29 (1989), 153-166.

[20] R. Deszcz, On Ricci-pseudosymmetric warped products, Demonstratio Math. 22 (1989), 1053-1065.

[21] R. Deszcz, On conformally flat Riemannian manifolds satisfying certain curvature conditions, Tensor (N.S.) 49 (1990), 134-145.

[22] R. Deszcz, On four-dimensional warped product manifolds satisfying certain pseudosymmetry curvature conditions, Colloq. Math. 62 (1991), 103-120.

[23] R. Deszcz, On pseudosymmetric spaces, Bull. Soc. Math. Belg. 44 (1992), Ser. A, 1-34.

[24] R. Deszcz, On pseudosymmetric warped product manifolds, in: Geometry and Topology of Submanifolds, V, World Sci., River Edge, NJ, 1993, 132-146.

[25] R. Deszcz, On some Akivis-Goldberg type metrics, Publ. Inst. Math. (Beograd) (N.S.) 74 (88) (2003), 71-83.

[26] R. Deszcz and M. Głogowska, Examples of nonsemisymmetric Ricci-semisymmetric hypersurfaces, Colloq. Math. 94 (2002), 87-101.

[27] R. Deszcz and M. Głogowska, Some nonsemisymmetric Ricci-semisymmetric warped product hypersurfaces, Publ. Inst. Math. (Beograd) (N.S.) 72 (86) (2002), 81-93.

[28] R. Deszcz, M. Głogowska, H. Hashiguchi, M. Hotloś and M. Yawata, On semi-Riemannian manifolds satisfying some conformally invariant curvature condition, Colloq. Math. 131 (2013), 149-170.

[29] R. Deszcz, M. Głogowska, M. Hotloś, and K. Sawicz, A Survey on Generalized Einstein Metric Conditions, Advances in Lorentzian Geometry: Proceedings of the Lorentzian Geometry Conference in Berlin, AMS/IP Studies in Advanced Mathematics 49, S.-T. Yau (series ed.), M. Plaue, A.D. Rendall and M. Scherfner (eds.), 2011, 27-46.

[30] R. Deszcz, M. Głogowska, M. Hotloś and Z. Șentürk, On certain quasi-Einstein semisymmetric hypersurfaces, Ann. Univ. Sci. Budapest. Eötvös Sect. Math. 41 (1998), 151-164.

[31] R. Deszcz, M. Głogowska, M. Hotloś and G. Zafindratafa, On some curvature conditions of pseudosymmetry type, Period. Math. Hung. 70 (2015), 153-170.

[32] R. Deszcz, M. Głogowska, M. Hotloś and G. Zafindratafa, Hypersurfaces in space forms satisfying some curvature conditions, to appear.

[33] R. Deszcz, M. Głogowska, J. Jełowicki, M. Petrović-Torgašev and G. Zafindratafa, On Riemann and Weyl compatible tensors, Publ. Inst. Math. (Beograd) (N.S.) 94 (108) (2013), 111-124.

[34] R. Deszcz, M. Głogowska, J. Jełowicki, M. Petrović-Torgašev and L. Verstraelen, Manifolds satisfying some special curvature conditions, to appear.

[35] R. Deszcz, M. Głogowska, M. Petrović-Torgašev and L. Verstraelen, On the Roter type of Chen ideal submanifolds, Results. Math. 59 (2011), 401-413.

[36] R. Deszcz, M. Głogowska, M. Petrović-Torgašev and L. Verstraelen, Curvature properties of some class of minimal hypersurfaces in Euclidean spaces, Filomat 29 (2015), 479-492.

[37] R. Deszcz, M. Głogowska, M. Plaue, K. Sawicz and M. Scherfner, On hypersurfaces in space forms satisfying particular curvature conditions of Tachibana type, Kragujevac J. Math. 35 (2011), 223-247.

[38] R. Deszcz and W. Grycak, On some class of warped product manifolds, Bull. Inst. Math. Acad. Sinica, 15 (1987), 311-322.

[39] R. Deszcz, S. Haesen and L. Verstraelen, On natural symmetries, "Topics in Differential Geometry", Ch. 6, Editors A. Mihai, I. Mihai and R. Miron, Editura Academiei Române, 2008.

[40] R. Deszcz, A.H. Hasmani, V.G. Khambholja and A.A. Shaikh, Curvature properties of interior black hole metric, arXiv:1401.6256v1 [math.DG], 24 Jan 2014.

[41] R. Deszcz and M. Hotloś, On certain subclass of pseudosymmetric manifolds, Publ. Math. Debrecen 53 (1998), 29-48.

[42] R. Deszcz and M. Hotloś, On hypersurfaces with type number two in spaces of constant curvature, Ann. Univ. Sci. Budapest. Eötvös Sect. Math. 46 (2003), 19-34. 
[43] R. Deszcz, M. Hotloś, J. Jełowicki, H. Kundu, and A.A. Shaikh, Curvature properties of Gödel metric, Int. J. Geom. Meth. Modern Phys. 11 (2014), 1450025 (20 pages).

[44] R. Deszcz, M. Hotloś, and Z. Șentürk, On some family of generalized Einstein metric curvature conditions, Demonstr. Math. 34 (2001), 943-954.

[45] R. Deszcz, M. Hotloś and Z. Șentürk, On the equivalence of the Ricci-pseudosymmetry and pseudosymmetry, Colloq. Math. 79 (1999), 211-227.

[46] R. Deszcz, M. Hotloś and Z. Șentürk, On curvature properties of certain quasi-Einstein hypersurfaces, Int. J. Math. 23 (2012), 1250073, 17 pp.

[47] R. Deszcz and D. Kowalczyk, On some class of pseudosymmetric warped products, Colloq. Math. 97 (2003), $7-22$.

[48] R. Deszcz and M. Kucharski, On curvature properties of certain generalized Robertson-Walker spacetimes, Tsukuba J. Math. 23 (1999), 113-130.

[49] R. Deszcz, M. Petrović-Torgašev, L. Verstraelen and G. Zafindratafa, On the intrinsic symmetries of Chen ideal submanifolds, Bull. Transilvania Univ. Brasov, Ser. III: Math. Informat., Phys., 1 (50) (2008), 99-108.

[50] R. Deszcz, M. Petrović-Torgašev, L. Verstraelen and G. Zafindratafa, On Chen ideal submanifolds satisfying some conditions of pseudo-symmetry type, Bull. Malaysian Math. Sci. Soc., in print.

[51] R. Deszcz, M. Plaue and M. Scherfner, On Roter type warped products with 1-dimensional fibres, J. Geom. Phys. 69 (2013), 1-11.

[52] R. Deszcz and M. Scherfner, On a particular class of warped products with fibres locally isometric to generalized Cartan hypersurfaces, Colloq. Math. 109 (2007), 13-29.

[53] R. Deszcz, P. Verheyen and L. Verstraelen, On some generalized Einstein metric conditions, Publ. Inst. Math. (Beograd) (N.S.) 60 (74) (1996), 108-120.

[54] R. Deszcz and L. Verstraelen, Hypersurfaces of semi-Riemannian conformally flat manifolds, in: Geometry and Topology of Submanifolds, III, World Sci., River Edge, NJ, 1991, 131-147.

[55] R. Deszcz, L. Verstraelen and L. Vrancken, The symmetry of warped product spacetimes, Gen. Relativ. Gravit. 23 (1991), 671-681.

[56] R. Deszcz, L. Verstraelen and S.. Yaprak, Warped products realizing a certain condition of pseudosymmetry type imposed on the Weyl curvature tensor, Chinese J. Math. 22 (1994), 139-157.

[57] R. Deszcz, L. Verstraelen and S. Yaprak, On 2-quasi-umbilical hypersurfaces in conformally flat spaces, Acta Math. Hungarica 78 (1998), 45-57.

[58] R. Deszcz and S.. Yaprak, Curvature properties of certain pseudosymmetric manifolds, Publ. Math. Debrecen 45 (1994), 333-345.

[59] M. Głogowska, Curvature conditions on hypersurfaces with two distinct principal curvatures, in: Banach Center Publ. 69, Inst. Math. Polish Acad. Sci., 2005, 133-143.

[60] M. Głogowska, On Roter type manifolds, in: Pure and Applied Differential Geometry - PADGE 2007, Shaker Verlag, Aachen, 2007, 114-122.

[61] M. Głogowska, On quasi-Einstein Cartan type hypersurfaces, J. Geom. Phys. 58 (2008), 599-614.

[62] K. Gödel, An example of a new type of cosmological solutions of Einsteins field equations of gravitation, Rev. Mod. Phys. 21 (1949) 447-450.

[63] J.B. Griffiths and J. Podolský, Exact Space-Times in Einsteins General Relativity, Cambridge Univ. Press, 2009.

[64] S. Haesen and L. Verstraelen, Natural intrinsic geometrical symmetries, Symmetry, Integrability and Geometry: Methods and Applications (SIGMA) 5 (2009), 086, 14 pp. Special Issue Elie Cartan and Differential Geometry".

[65] G. Hall, Projective Structure in Differential Geometry, Proceedings of the International Conference XVI Geometrical Seminar, September, 20-25, Vrnjačka banja, September, 20-25, 2010, Faculty of Science and Mathematics, University of Niš, Serbia, 2011, 40-55.

[66] G. Hall and Z. Wang, Projective structure in 4-dimensional manifolds with positive definite metrics, J. Geom. Phys. 62 (2012), 449-463.

[67] A. Hasni, Les géométries de Thurston et la pseudo-symétrie d'apreès R. Deszcz, Thèse de doctorat en mathématique, Université Abou Bakr Belkaid-Tlemcen, Faculté de Sciences, Département de Mathématiques, 2014. 
[68] D. Kowalczyk, On some class of semisymmetric manifolds, Soochow J. Math. 27 (2001), $445-461$.

[69] D. Kowalczyk, On the Reissner-Nordström-de Sitter type spacetimes, Tsukuba J. Math. 30 (2006), $363-381$.

[70] O. Kowalski, A classification of Riemannian 3-manifolds with constant principal Ricci curvatures $\rho_{1}=$ $\rho_{2} \neq \rho_{3}$, Nagoya Math. J. 132 (1993), 1-36.

[71] G.I. Kruchkovich, On semi-reducible Riemannian spaces (in Russian), Dokl. Akad. Nauk SSSR 115 (1957), $862-865$.

[72] G.I. Kruchkovich, On some class of Riemannian spaces (in Russian), Trudy sem. po vekt. i tenz. analizu 11, (1961), 103-128.

[73] G.I. Kruchkovich, On spaces of V.F. Kagan (in Russian), in: V.F. Kagan, Subprojective spaces, Moscow, 1961, 163-198.

[74] H. Li, L. Vrancken, X. Wang, A new characterization of the Berger sphere in complex projective space, J. Geom. Phys. 92 (2015), 129-139.

[75] M.A. Magid, Indefinite Einstein hypersurfaces with imaginary principal curvatures, Houston J. Math. 10 (1984), 57-61.

[76] B. O'Neill, Semi-Riemannian Geometry with Applications to Relativity, Academic Press, New York - London, 1983.

[77] K. Sawicz, Hypersurfaces in spaces of constant curvature satisfying some Ricci-type equations, Colloq. Math. 101 (2004), 183-201.

[78] K. Sawicz, On curvature characterization of some hypersurfaces in spaces of constant curvature, Publ. Inst. Math. (Beograd) (N.S.) 79 (93) (2006), 95-107.

[79] K. Sawicz, Curvature properties of some class of hypersurfaces in Euclidean spaces, Publ. Inst. Math. (Beograd) (N.S.), in print.

[80] J.M.M. Senovilla and R. Torres, Particle production from marginally trapped surfaces of general spacetimes, Class. Quantum Grav. 32 (2015) 085004 (18pp).

[81] A.A. Shaikh, R. Deszcz, M. Hotloś, J. Jełowicki and H. Kundu, On pseudosymmetric manifolds, Publ. Math. Debrecen, in print.

[82] A.A. Shaikh and H. Kundu, On generalized Roter type manifolds, arXiv: 1411.0841v1 [math.DG] 4 Nov 2014.

[83] A.A. Shaikh and H. Kundu, On warped product generalized Roter type manifolds, arXiv: 1411.0845v1 [math.DG] 4 Nov 2014.

[84] H. Stephani, D. Kramer, M. Maccallum, C. Hoenselaers and E. Herlt, Exact Solutions of Einstein's Field Equations, second ed. Cambridge Monographs on Mathematical Physics, Cambridge U.P., 2003.

[85] Z.I. Szabó, Structure theorems on Riemannian spaces satisfying $R(X, Y) \cdot R=0$. I. The local version, J. Differential Geom. 17 (1982), 531-582.

[86] Y. Tashiro, Compact four-dimensional Einstein manifolds, J. Differential Geom. 16 (1981), 475-481.

[87] P.C. Vaidya, The external field of a radiating star in general relativity, Current Sci. (India) 12 (1943), 183.

[88] L. Verstraelen, A concise mini history of geometry, Kragujevac J. Math. 38 (2014), 5-21.

Ryszard Deszcz, Małgorzata Głogowska and Jan Jełowicki

Department of Mathematics

Wrocław University of Environmental and Life Sciences

Grunwaldzka 53, 50-357 Wrocław, Poland

E-mail: Ryszard.Deszcz@up.wroc.pl E-mail: Malgorzata.Glogowska@up.wroc.pl E-mail: Jan.Jełowicki@up.wroc.pl

Georges Zafindratafa

Laboratoire de Mathématiques et Applications de Valenciennes

Université de Valenciennes et du Hainaut-Cambrésis

Le Mont Houy, Lamath-ISTV2, 59313 Valenciennes Cedex 9, France

E-mail: Georges.Zafindratafa@univ-valenciennes.fr 